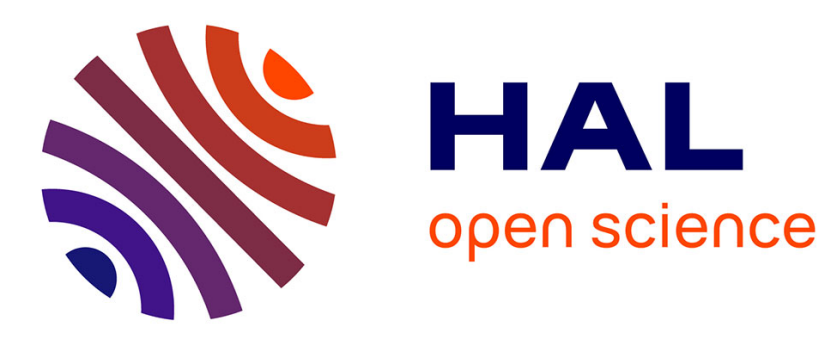

\title{
Hierarchical Sticker and Sticky Chain Dynamics in Self-Healing Butyl Rubber Ionomers
}

\author{
Anton Mordvinkin, Marcus Suckow, Frank Boḧme, Ralph H Colby, \\ Costantino Creton, Kay Saalwächter
}

\section{- To cite this version:}

Anton Mordvinkin, Marcus Suckow, Frank Bö̈me, Ralph H Colby, Costantino Creton, et al.. Hierarchical Sticker and Sticky Chain Dynamics in Self-Healing Butyl Rubber Ionomers. Macromolecules, 2019, 52 (11), pp.4169-4184. 10.1021/acs.macromol.9b00159 . hal-02396313

\section{HAL Id: hal-02396313 \\ https://hal.science/hal-02396313}

Submitted on 5 Dec 2019

HAL is a multi-disciplinary open access archive for the deposit and dissemination of scientific research documents, whether they are published or not. The documents may come from teaching and research institutions in France or abroad, or from public or private research centers.
L'archive ouverte pluridisciplinaire HAL, est destinée au dépôt et à la diffusion de documents scientifiques de niveau recherche, publiés ou non, émanant des établissements d'enseignement et de recherche français ou étrangers, des laboratoires publics ou privés. 


\title{
Hierarchical Sticker and Sticky Chain Dynamics in Self-Healing Butyl Rubber lonomers
}

\author{
Anton Mordvinkin, ${ }^{\dagger}$ Marcus Suckow, ${ }^{\ddagger}$ Frank Böhme, ${ }^{\ddagger \odot}$ Ralph H. Colby, ${ }^{\S}$ Costantino Creton, ${ }^{\|(\odot)}$ \\ and Kay Saalwächter, ${ }^{* \dagger \odot}$ \\ ${ }^{\dagger}$ Institut für Physik-NMR, Martin-Luther-Universität Halle-Wittenberg, Betty-Heimann-Str. 7, 06120 Halle (Saale), Germany \\ ${ }^{\ddagger}$ Leibniz-Institut für Polymerforschung Dresden e.V., Hohe Straße 6, 01069 Dresden, Germany \\ ${ }^{\S}$ Materials Science and Engineering, The Pennsylvania State University, University Park, Pennsylvania 16802, United States \\ "Laboratoire de Sciences et Ingénierie de la Matière Molle, CNRS, ESPCI Paris, PSL Research University, 10 Rue Vauquelin, 75005 \\ Paris, France
}

ABSTRACT: We present a detailed comparison of the microscopic dynamics and the macroscopic mechanical behavior of novel butyl rubber ionomers with tunable dynamics of sparse sticky imidazole-based sidegroups that form clusters of about 20 units separated by essentially unperturbed chains. This material platform shows promise for application as self-healing elastomers. Size and thermal stability of the ionic clusters were probed by small-angle X-ray scattering, and the chain and sticker dynamics were studied by a combination of broadband dielectric spectroscopy (BDS) and advanced NMR methods. The results are correlated with the rheological behavior characterized by dynamicmechanical analysis (DMA). While the NMR-detected chain relaxation and DMA results agree quantitatively and confirm relevant aspects of the sticky-reptation picture on a microscopic level, we stress and explain that apparent master curves are of limited use for such a comparison. The cluster-related relaxation time detected by BDS is much shorter than the elastic chain relaxation time, although the weak conductivity does follow the latter. The systematic trends across the sample series suggest that all relaxations are dominated by a cluster-related activation barrier, but also that the BDS-based cluster relaxation does not seem to be directly associated with the effective sticker lifetime. Nonlinear stress-strain experiments demonstrate a reduction of sticker lifetime on stretching and that the stored stress and the elastic recovery depend on the deformation rate.

\section{INTRODUCTION}

Since the first ionic cross-linking of carboxylated polymers in the 1950s, ${ }^{1}$ ionomers have been valued for their exceptional mechanical properties, ${ }^{2-4}$ melt processability, ${ }^{5}$ recyclability, ${ }^{6,7}$ and also their self-healing ability. ${ }^{3,4,8,9}$ These material properties are connected to the ability of ionic groups to reversibly associate and form ionic aggregates, ${ }^{2}$ which are stable at operating temperatures and become labile as the temperature increases. ${ }^{10}$ The ionic aggregation features a hierarchy of structures formed: starting from the association of two contact ion pairs to ionic multiplets, we have further aggregation into larger phase-separated regions, called ionic clusters, which also comprise immobilized polymer chains with their own glasstransition temperature $\left(T_{\mathrm{g}}\right){ }^{11-14}$ The final morphology depends on various factors such as polarity of the polymer matrix, its flexibility, $T_{g}$, nature and size of pendant ions and their counterions, distribution of ions along the polymer backbone, ion content, presence of moisture residues, thermal history, etc. ${ }^{10,13-15}$ As a result, the properties of ionomers can be adapted by variation of the above parameters. The objects of the present investigation are bromobutyl rubbers (bromi- nated isobutylene-isoprene rubber, BIIR) modified with alkylimidazolium (AI) moieties ${ }^{16}$ exhibiting favorable selfhealing capacity, as reported very recently., ${ }^{3,4,17}$ Importantly, their association lifetime (henceforth referred to as sticker lifetime, $\left.\tau_{\mathrm{st}}^{*}\right)$ and thus the material properties can be tuned by variation of the alkyl side chain of the imidazole unit. ${ }^{4}$ This provides the basis of a systematic study presented herein, allowing for qualitatively new insights.

Autonomous self-healing relies on the possibility of chain motion across a damaged area and re-formation of physical links among the stickers. ${ }^{18}$ The latter can be realized by the above-mentioned ionic associations, but also through hydrogen bonding and $\pi-\pi$ interactions of uncharged moieties. The most established model describing sticky chain dynamics is the sticky-reptation model, ${ }^{19}$ where a binary sticker lifetime $\tau_{\mathrm{st}}^{*}$ is taken to govern the long-time (low-frequency) rheological behavior; see Figure 1a. While the underlying tube/reptation 

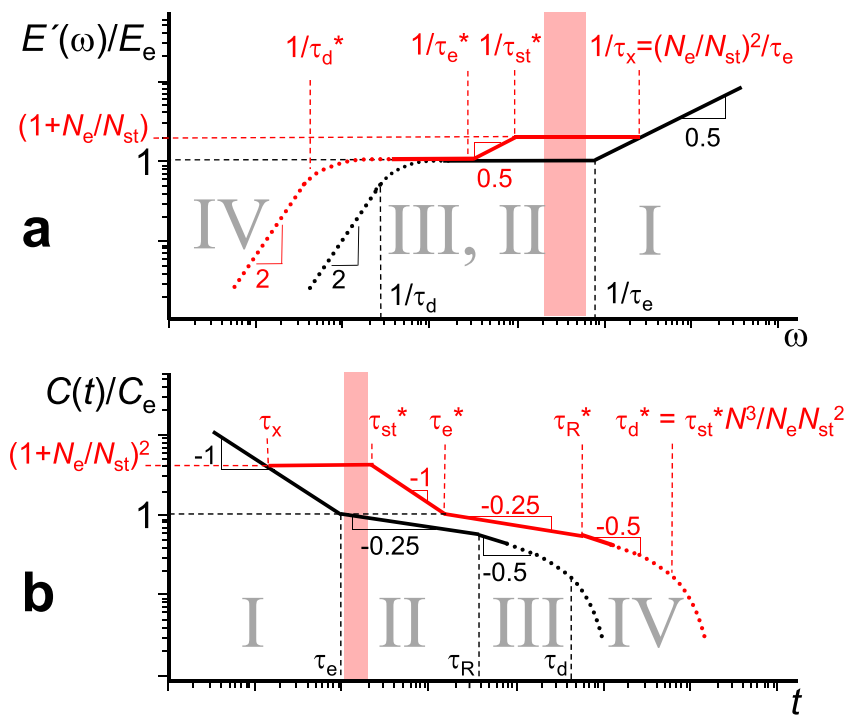

Figure 1. Dynamic regimes (Roman background numerals) and characteristic crossover times for an entangled polymer melt (tube model, black) and a dynamic network (sticky reptation, red) with $N_{\mathrm{e}} /$ $N_{\text {st }} \geq 1$ (number $N_{\mathrm{e}}$ segments per entangled strand and $N_{\text {st }}$ segments per sticker) for the (a) storage modulus $E^{\prime}(\omega)^{19,21}$ and (b) segmental orientation autocorrelation function $C(t),{ }^{43}$ both normalized to the corresponding quantity at the level of entanglements. The dotted parts are not accessed in the present study. Shaded boxes mark the transition between temperature dependencies due to segmental dynamics and sticker activation. The latter are marked with asterisks for the characteristic times $\tau$.

model $^{20}$ predicts an entanglement-related plateau modulus spanning the constrained Rouse (II) and reptation regimes (III) between the inverse terminal $\left(1 / \tau_{\mathrm{d}}\right)$ and entanglement times $\left(1 / \tau_{\mathrm{e}}\right)$, stickers lead to an increased "Leibler" plateau modulus extending to a correspondingly higher transition frequency $1 / \tau_{\mathrm{x}}$. A narrow sticky Rouse regime extending down to the inverse sticky entanglement time $1 / \tau_{\mathrm{e}}^{*}$ marks its lowfrequency end, below which the entanglement-only plateau extends to the inverse sticky terminal time $1 / \tau_{\mathrm{d}}^{*}$; both of these time scales are governed by $\tau_{\mathrm{st}}^{*}$ as the elementary process. Applications of this model to explain rheological data require extensions taking into account interchain interactions via, for example, double reptation. ${ }^{21}$ Alternatives include slip-link models ${ }^{22,23}$ or an algorithmic implementation of the tube model, ${ }^{24}$ which allows for the additional consideration of hindered fluctuations along the tube. The latter two works emphasize the possibility of lumping the sticker effect into an increased, effective friction coefficient.

Potential complications arise when the sticky groups form clusters rather than pairwise aggregates, ${ }^{21,25-30}$ where entropic effects related to the formation of "flowerlike micelles" may come into play. $^{29}$ An extensive treatment by the scaling theory $^{29}$ suggests a decoupling of chain-level relaxation and terminal flow, the latter being dominated by micellar rearrangement. In turn, simulations ${ }^{30}$ stress the relevance of a partner-exchange mechanism among micelles. A critical assessment seems to be open, as many even recent investigations of systems with actually or potentially aggregate-forming stickers have not taken this into account. Avoiding the related ambiguities, we here focus on times shorter than the disentanglement time $\tau_{\mathrm{d}}$ (see the solid line sections in Figure 1), also owing to the fact that our ionomers are based upon a polydisperse high-molecular-weight (MW) industrial BIIR and decompose at $T>140{ }^{\circ} \mathrm{C}$.

It was suggested that the temperature dependence of the sticker lifetime ${ }^{18,29,31-33}$

$$
\tau_{\mathrm{st}}^{*}(T)=\tau_{0}(T) \mathrm{e}^{E_{\mathrm{a}}^{\mathrm{st}} / R T}
$$

is governed by an activation energy $E_{\mathrm{a}}^{\text {st }}$ that comprises an actual barrier and a binding energy. The attempt time $\tau_{0}(T)$ is associated with the time scale of segmental motion $\tau_{\alpha}(T)^{18,29,31}$ and thus the segmental friction coefficient $\zeta(T)$, which typically follows a Vogel-Fulcher (VF) behavior. In a limited $T$ range, the apparent activation energy $E_{\mathrm{a}}^{\mathrm{app}}(T)$ obtained for $\tau_{\mathrm{st}}^{*}(T)$ is thus the sum of $E_{\mathrm{a}}^{\text {st }}$ and $E_{\mathrm{a}}^{\alpha}(T)$, the latter representing the approximate Arrhenius $T$ dependence of $\tau_{\alpha}(T) \propto \mathrm{e}^{E_{\mathrm{a}}^{\alpha}(T) / R T} . E_{\mathrm{a}}^{\text {st }}$ is commonly assumed to be constant, whereas $E_{\mathrm{a}}^{\alpha}(T)$ can be treated as constant in a sufficiently narrow temperature interval. The transition of the rheological behavior from segment-based VF behavior (up to the entanglement time $\tau_{\mathrm{e}}$ ) to a stronger $T$ dependence for motions on time scales around and beyond $\tau_{\mathrm{st}}^{*}(T)$, marked by the shaded boxes in Figure 1, thus leads to complications in applying time-temperature superposition (TTS) $)^{34,35}$ to construct rheological master curves, as discussed in many publications, for example, refs 36-38. Below, we critically reflect the use of such apparent master curves when comparing results from different methods.

The sticky-reptation model was later refined to include a renormalization of $\tau_{\mathrm{st}}^{*}$ to account for returns to the original binding site, ${ }^{32}$ and a more accurate treatment published in the context of a theory of self-healing ${ }^{18}$ was recently shown ${ }^{39,40}$ to be able to account for a potentially large deviation between $\tau_{\mathrm{st}}^{*}$ determined by rheology and microscopic measurements of potentially related time scales by, for example, broadband dielectric spectroscopy (BDS). ${ }^{36,39-42}$ The latter directly probes reorientations of molecular dipoles associated with the stickers, whereas the rheology is sensitive to reorientations of whole chain segments. Notably, the deviation between the two methods has been observed to become negligible at high sticker fractions ${ }^{36,37,41}$ likely because returns become less probable at higher aggregate fractions. We will revisit this question below and add for the first time an experimental perspective on the microscopic chain dynamics as probed by solid-state NMR.

Solid-state NMR represents a powerful complement to BDS, with different methods providing access to the dynamics of sticky (e.g., hydrogen-bonded) groups with chemical resolution $^{44}$ or chain dynamics in entangled polymers across all regimes of the tube model. ${ }^{43,45-49}$ We here use ${ }^{1} \mathrm{H}$ doublequantum (DQ) NMR under magic-angle spinning (MAS) conditions $^{44,50}$ to directly complement BDS data to assess the sticker dynamics and apply low-resolution static ${ }^{1} \mathrm{H}$ DQ $\mathrm{NMR}^{43,51-53}$ to assess the sticky chain dynamics. The latter probes the segmental orientation autocorrelation function (OACF) of the second Legendre polynomial $C(t)=$ $5\left\langle P_{2}(\cos \theta(t)) P_{2}(\cos \theta(0))\right\rangle,{ }^{48}$ where $\theta$ is the segmental orientation with respect to an external reference direction. This makes it comparable to rheological experiments, which at lower frequencies probe the orientation correlations of a Rouse segment. In Figure $1 \mathrm{~b}$, we depict the form of $C(t)$ featuring different power laws characteristic for the above-mentioned tube-model regimes. Note that the similarity of the shapes of $C(t)$ and the stress relaxation modulus, $E(t)$ or $G(t)$, is not a 
coincidence but rather the consequence of their similar theoretical treatments. ${ }^{43,45,46}$

In comparison to $E^{\prime}(\omega)$ shown in Figure 1a, the OACF has an additional sensitivity to the regime II-III transition at the Rouse time $\tau_{\mathrm{R}}$, below and above which the reptation model predicts power-law exponents (log-log slopes) $\kappa$ of 0.25 and 0.5 , respectively. ${ }^{45}$ In regimes I and II, $\kappa$ is experimentally found to deviate from the predictions, assuming values on the range of $0.7-0.9$ and $0.29-0.5$, respectively, the latter depending on $\mathrm{MW} \cdot{ }^{43,47-49}$ For transient networks, starting from the Rouse time of a chain section between two stickers $\tau_{x}$, one expects the mentioned Leibler plateau with a slope approaching zero, as no long-range dynamics occurs. It again ends with a short period of sticky Rouse motion, ${ }^{21}$ followed by the later regimes with delayed regime transitions that are all slaved to $\tau_{\text {st }}^{*}$.

Our main focus is the quantification of the microscopic sticker and sticky chain dynamics in the above-mentioned BIIR-based ionomers by MAS and static DQ NMR, respectively, and their correlation with corresponding results from BDS and dynamic-mechanical analysis (DMA). We complement our study by small-angle X-ray scattering (SAXS) to explore the structure of ionic clusters and finally apply nonlinear tensile tests to assess the dependence of the studied time scales on external and internal stress.

\section{EXPERIMENTAL SECTION}

II.I. Samples. Bromobutyl rubber (BIIR) from Lanxess was studied in the as-received (un-cross-linked) state as well as after vulcanization either with a conventional sulfur-based recipe to obtain covalently cross-linked samples or by means of introducing ionic alkylimidazolium bromide moieties at the brominated sites to obtain supramolecular ionic networks. ${ }^{16}$ The modification reactions were performed with methyl-, butyl-, hexyl, or nonylimidazole in the bulk melt, resulting in samples denoted BIIR+MI, $+\mathrm{BI},+\mathrm{HI}$, and $+\mathrm{NI}$, respectively (see Figure 2, top)., ${ }^{3,4}$ To this end, alkylimidazoles were added in an excess of 1.5:1 with respect to brominated isoprene units having a molar fraction of $x_{\mathrm{i}}=0.8 \mathrm{~mol} \%$. The noted fraction and the

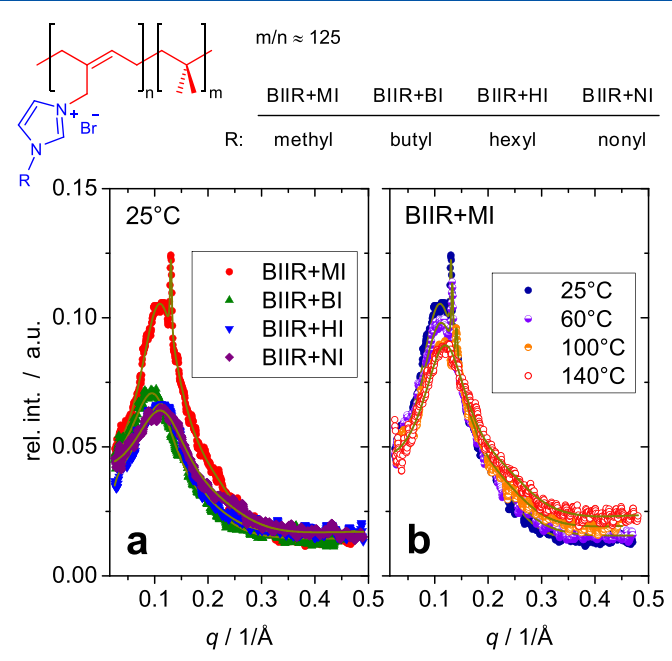

Figure 2. (a) Comparison of SAXS data of all ionic networks at 25 ${ }^{\circ} \mathrm{C}$. (b) SAXS data of BIIR+MI at the indicated temperatures. The solid lines are the fits according to a model based upon a PercusYevick structure factor of hard-sphere-like micellar aggregates with a scattering core. ${ }^{26,28,56}$ The chemical structure of the samples is sketched at the top. full conversion were assessed by ${ }^{1} \mathrm{H}$ solution-state NMR, monitoring the main-chain resonances. ${ }^{4}$

The molecular weight $\left(M_{\mathrm{w}}\right)$ of the base BIIR was estimated to about $590 \mathrm{~kg} / \mathrm{mol}$ (PDI $\approx 4.7$ ), as measured by size exclusion chromatography on an Agilent series 1100 high-performance liquid chromatography apparatus equipped with an Agilent PL Mixed-B-LS column $\left(300 \times 7.5 \mathrm{~mm}^{2}, 5 \mu \mathrm{m}\right.$ polystyrene (PS) gel) calibrated against polystyrene standards at a flow rate of $1 \mathrm{~mL} / \mathrm{min}$ in $\mathrm{CHCl}_{3}$. With a monomer molecular weight $M_{\text {mon }}$ of $56 \mathrm{~g} / \mathrm{mol}$ and the number of monomers between two imidazolium stickers of $N_{\mathrm{st}}^{\mathrm{mon}}=1 / x_{i}=125$, we have transient networks with a molecular weight between stickers $M_{\mathrm{st}}$ of $7 \mathrm{~kg} / \mathrm{mol}$ and thus about 85 stickers per chain. With a Kuhn segment molecular weight of 274 and an entanglement molecular weight $M_{\mathrm{e}}$ of $6.7 \mathrm{~kg} / \mathrm{mol}$ at room temperature, ${ }^{54}$ we obtain $N_{\mathrm{e}} \approx 25$ versus $N_{\text {st }}=26 \mathrm{Kuhn}$ segments between entanglements versus stickers, respectively, and a ratio of $N_{\mathrm{e}} / N_{\mathrm{st}} \approx 1$.

All samples were prepared in the form of press-molded sheets of about 1 or $2 \mathrm{~mm}$ thickness. For high-resolution MAS NMR and BDS measurements, the unreacted alkylimidazole species were removed by reprecipitation. This is necessary to avoid the related signal contributions to focus only on the stickers and not on the free imidazole. For low-resolution NMR and most mechanical experiments, the samples were studied as prepared, but consistency of the results obtained with these two techniques with precipitated counterparts was confirmed on a few examples. Covalent networks with different cross-link densities were prepared using $x=0.5$ and 1 parts per hundred rubber sulfur (denoted BIIR-S $x$ ). It is stressed that the ionic modification did not increase $T_{\mathrm{g}}$ of the polymer matrix, as is most obvious from the high-frequency mechanical properties reported below. BIIR-S1 had to be additionally thermally treated at $140{ }^{\circ} \mathrm{C}$ for $2 \mathrm{~h}$ in a protective argon atmosphere to guarantee the reaction of all added sulfur. Degradation processes occurred at temperatures beyond $140{ }^{\circ} \mathrm{C}$ in all modified BIIR samples, thus restricting our experimental $T$ range.

II.II. Small-Angle X-ray Scattering. SAXS measurements were carried out in a temperature range from 25 up to $140^{\circ} \mathrm{C}$ on a Xenocs Xeuss 2.0 setup equipped with a Linkam hotstage and a Pilatus twodimensional detector in the virtual detection mode for the suitable $q$ range, obtaining one-dimensional (1D) traces via weighted azimuthal averaging. Before the measurements, the samples were annealed at $140{ }^{\circ} \mathrm{C}$ in vacuum for $24 \mathrm{~h}$. The sample thickness of around $1 \mathrm{~mm}$ was carefully controlled, and the scattered intensity was corrected for thickness variations by way of transmission experiments at $q=0$. In this way, the scattering intensities of all samples can be compared and interpreted in terms of differences in scattering contrast.

II.III. Solid-State NMR. Low-resolution, time-domain NMR experiments were performed on Bruker Minispec mq20 benchtop spectrometers operating at a Larmor frequency of about $20 \mathrm{MHz}\left(B_{0}\right.$ $=0.47 \mathrm{~T}$ ), with 90 and $180^{\circ}$ pulses of 1.6 and 2.8 or 3.3 and $5.2 \mu \mathrm{s}$ and a dead time of 14 or $12 \mu$ s, respectively. The samples in the form of $\varnothing 8 \mathrm{~mm}$ disks were placed into $10 \mathrm{~mm}$ o.d. NMR tubes, which were filled with argon and flame-sealed to prevent degradation. Highresolution MAS NMR experiments at $10 \mathrm{kHz}$ spinning were carried out on a Bruker Avance III spectrometer $\left(B_{0}=9.4 \mathrm{~T}\right)$ with a tripleresonance $4 \mathrm{~mm}$ MAS probe. The samples were put inside sealable Kel-F inserts, which were then introduced into $\mathrm{ZrO}_{2}$ rotors and closed by Vespel caps. In this case, the $90^{\circ}$ pulse was set to $3 \mu$ s and the $180^{\circ}$ pulse to twice this value. To isolate the imidazolium signals from the overlapping aliphatic signals of much larger intensity, the latter were considered as an increasing baseline in the higher-ppm spectral range around the imidazolium signals and subtracted subsequently. The acquired NMR spectra were referenced by setting the methyl signal to the known chemical shift determined by solution-state NMR. For all NMR experiments, the experimental temperature was controlled by a Bruker BVT 3000 air control unit with an accuracy of around $1 \mathrm{~K}$.

II.IV. Dynamic-Mechanical Analyses. DMA was performed on a Norleq Q800 instrument, recording frequency sweeps from 0.3 to $30 \mathrm{~Hz}$ within a temperature range between -50 and $140{ }^{\circ} \mathrm{C}$ with $5{ }^{\circ} \mathrm{C}$ steps, allowing for $3 \mathrm{~min}$ of equilibration each. A linear response was assured by aid of stress-strain curves. Master curves were constructed 
on the basis of $\tan \delta$ data sets to obtain the horizontal shift factors. The storage and loss moduli ( $E^{\prime}$ and $E^{\prime \prime}$, respectively) at different temperatures were then corrected by multiplication with the entropic factor $T_{\text {ref }} / T$ ( $T_{\text {ref }}$ is the reference temperature), after which vertical shift factors $b_{\mathrm{T}}$ were additionally applied to obtain data overlap at different temperatures. The main factors influencing the $b_{\mathrm{T}}$ were small changes in sample thickness arising from anisotropic thermal expansion possibly due to a weak memory effect in the press-molded samples.

Tensile experiments in the linear and nonlinear viscoelastic regimes were performed on an Instron 5565 tensile tester with a $100 \mathrm{~N}$ load cell at room temperature. The specimens were prepared in the form of rectangular sheets with 4 or $5 \mathrm{~mm}$ width, $\sim 1 \mathrm{~mm}$ thickness, and $\sim 20$ $\mathrm{mm}$ length. Changes in length were monitored using a video extensometer. Cyclic uniaxial stress-strain experiments were performed with loading-unloading cycles in four steps until about $50,100,150$, and $200 \%$ of strain with a strain rate $(\dot{\epsilon})$ of $0.02 \mathrm{~s}^{-1}$. Stress relaxation experiments were conducted after small $(\leq 2 \%)$ and large $(\sim 200 \%)$ strain steps and at $21 \%$ strain after a loadingunloading cycle to $\sim 200 \%$ strain, all with $\dot{\epsilon}=0.1 \mathrm{~s}^{-1}$.

II.V. Broadband Dielectric Spectroscopy. BDS was used to probe dielectric functions from 0.1 to $10^{6} \mathrm{~Hz}$ at temperatures from -50 to $+140{ }^{\circ} \mathrm{C}$ using a Novocontrol $\alpha$ dielectric analyzer equipped with a Quatro Cryosystem employing evaporated liquid $\mathrm{N}_{2}$ as a cooling and heating agent, with a temperature precision of $0.1 \mathrm{~K}$. Disc-shaped specimens of about $16 \mathrm{~mm}$ diameter, punched from ca. 1 $\mathrm{mm}$-thick rubber sheets and vacuum-dried at $70{ }^{\circ} \mathrm{C}$ for several days, were press-molded for $\sim 30 \mathrm{~min}$ at $140{ }^{\circ} \mathrm{C}$ between disc-shaped polished brass electrodes (upper electrode diameter of $15 \mathrm{~mm}$ ), placing three Teflon spacers of $3 \mathrm{~mm}$ diameter in three smaller punched holes to ensure a final capacitor spacing of $0.75 \mathrm{~mm}$. See the Supporting Information (SI) for details.

\section{RESULTS AND DISCUSSION}

III.I. Ionic-Cluster Size and Separation from SAXS. We first focus on the presence and thermal stability of ionic aggregates present in the ionic networks. Figure 2a shows scattering profiles of all four ionic samples at room temperature. They all exhibit the typical "ionomer peak" at around 0.095-0.1 $\AA^{-1}$, the position of which is thus shown to be nearly invariant to the alkyl group length in the alkylimidazolium (AI) moieties. The only relevant difference among the samples is the lower relative intensity for the samples with higher-length alkyl residues, which can be attributed to a correspondingly lower average electron density, reducing the scattering contrast between the ionic clusters and the rubber matrix. The narrow peak at $q=0.13 \AA^{-1}$ for BIIR+MI is related to a residual amount of crystalline stearate that is part of the commercial formulation of BIIR, ${ }^{55}$ whereas its absence in the other samples may be related to a higher solubility of the stearate moiety in the ionic clusters. A more detailed discussion is deferred to the SI.

The observed $q$ value for the ionic correlation corresponds to an average distance between the ionic aggregates $r_{\mathrm{ii}}=2 \pi / q$ of $62.8 \AA$. Remarkably, the root-mean-square end-to-end distance corresponding to the part of the chain between two ionic cross-links $\sqrt{R^{2}}=\sqrt{C_{\infty} 2 N_{\mathrm{st}}^{\mathrm{mon}} a_{\mathrm{b}}^{2}} \approx 62 \AA$, where $C_{\infty}=$ 6.6 is the characteristic ratio of $\mathrm{PIB},{ }^{54} 2 \mathrm{~N}_{\mathrm{st}}^{\mathrm{mon}}=250$ is the number of bonds between two ionic cross-links, and $a_{\mathrm{b}}$ is the carbon-carbon bond length. ${ }^{57}$ Similar values of $r_{\mathrm{ii}}$ and $\sqrt{R^{2}}$ suggest that the ionic groups are mostly in the associated state and that cluster formation does not involve significant chain stretching.

The temperature dependence of the scattering curves up to $140{ }^{\circ} \mathrm{C}$ for the ionic networks BIIR+MI and BIIR+BI is shown in Figures $2 \mathrm{~b}$ and SI1b, respectively. We observe almost no change of the peaks' positions and an only weak intensity loss of around $20 \%$. A decrease in intensity can be caused by reduction of positional correlations, an acceleration of sticky dynamics of single ionic groups in the proximity of the aggregates, or a shift of the open/closed equilibrium toward the open, matrix-dissolved state. The overall weak changes suggest that the average distance between the ionic aggregates does not change and that the clusters stay largely intact (they may of course exchange stickers via a low concentration of free stickers).

Broad, featureless SAXS peaks can result from various disordered structures of different geometries. ${ }^{58-60}$ Therefore, we present two different estimations of aggregation numbers (ANs), that is, the average number of ionic groups per ionic aggregate. We can simply use a space-filling argument ${ }^{12,28}$ with the assumption that all of the ionic aggregates are arranged in a cubic lattice with the size of the unit cell equal to $r_{\mathrm{ii}}$. The aggregation number is then calculated as follows:

$$
\mathrm{AN}=r_{\mathrm{ii}}^{3}\left(\frac{M_{\mathrm{st}}}{\rho_{\mathrm{PIB}} N_{\mathrm{A}}}\right)^{-1} \approx 20 \pm 6
$$

where the density $\rho_{\text {PIB }}$ is about $0.9 \mathrm{~g} / \mathrm{cm}^{3},{ }^{54} M_{\text {st }} \approx 7 \mathrm{~kg} / \mathrm{mol}$ is the molecular weight of the part of the chain between two stickers, and $N_{\mathrm{A}}$ is Avogadro's number.

Alternatively, we can employ a model to fit the scattering curves, for which we use a previous approach ${ }^{26,28,56}$ based on the assumption of "flowerlike micelles" with hard-sphere repulsion, described by a Percus-Yevick structure factor and a much smaller spherical core providing the scattering contrast. Details are given in the SI; see in particular Table S1. Note that the relevant size of the ionic aggregates in this model is not encoded in the position of the ionomer peak but in the shape of its high- $q$ tail, which is governed by the decaying form factor of the spherical core.

Reassuringly, this approach provides an AN of about $22 \pm 9$, in very satisfactory agreement with the first, less modeldependent approach. The similarity of the cluster size across our sample series, with an $\mathrm{AN}$ of around 20, provides confirmation of the assumption that the overall structure is governed by the spacing of the sticky groups along the chain and by their strong aggregation tendency in all cases. We can use scaling arguments, ${ }^{29}$ adapted to ideal chains in bulk, to estimate the number of bridges $N_{\mathrm{br}}$ of a flowerlike micelle and thus the fraction of bridge- (vs loop-) forming stickers to

$$
N_{\text {br }} / \mathrm{AN} \sim\left(N_{\mathrm{st}}^{0.5} / \mathrm{AN}\right)^{0.22} \approx 0.74
$$

Combined with the above finding on the sticker separation, we conclude that the samples are dominated by bridging chains with close-to-ideal dimensions.

III.II. Ionic-Cluster Dynamics from BDS. Turning to dynamics, we first discuss the BDS results as they cover the widest frequency and temperature range, providing a framework for the NMR and DMA results. Exemplarily, the dielectric function (real and imaginary parts) for one of our samples $(\mathrm{BIIR}+\mathrm{HI})$ is shown in Figure 3a for two different temperatures. Fits have been performed simultaneously to $\epsilon^{\prime \prime}$ and $\epsilon^{\prime}$ using a combination of up to two Havriliak-Negami (HN) functions and a power law to account for conductivity; see ref 61 and the SI for details on the fitting and the assignment of the different relaxations. The $\mathrm{HN}$ fit provides a relaxation strength $\Delta \epsilon$, a relaxation time $\tau$ (which is a log-scale 


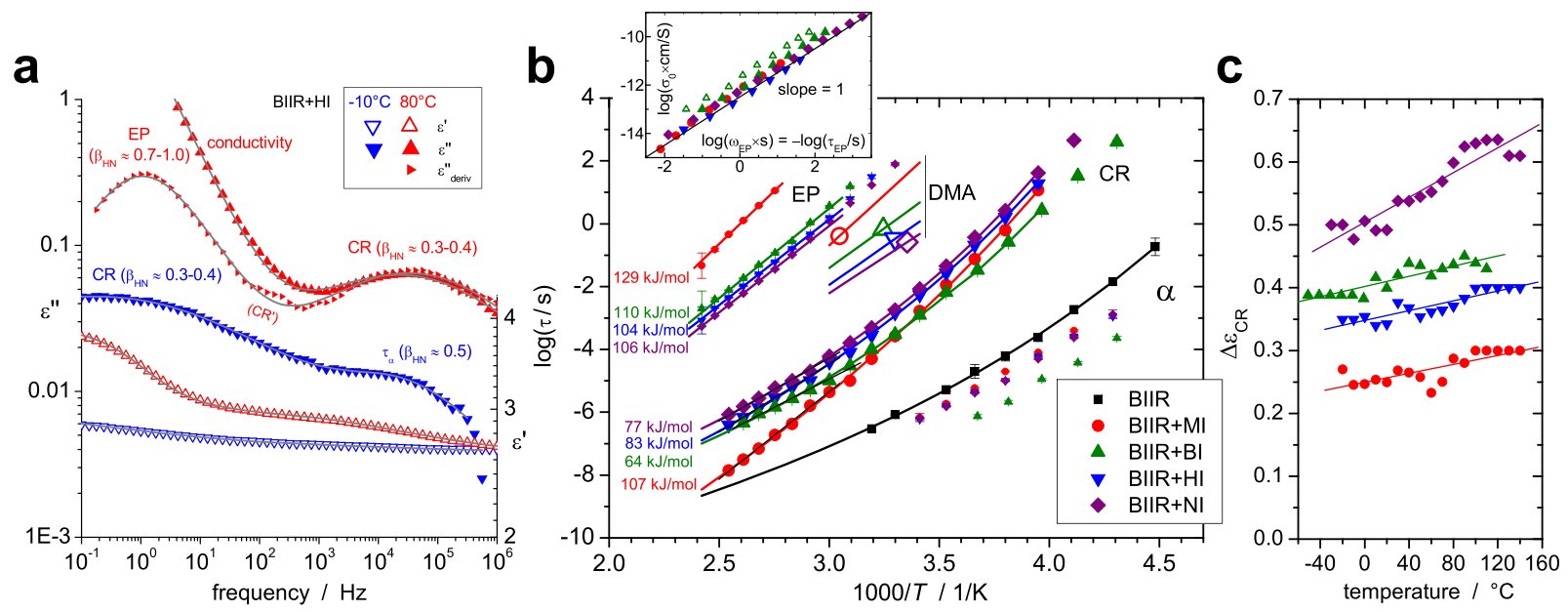

Figure 3. (a) Dielectric function for BIIR+HI at two different temperatures, including the derivative quantity $\epsilon^{\prime \prime}{ }_{\text {deriv }}$ (see the SI), with simultaneous $\mathrm{HN}$ fits to $\epsilon^{\prime}$ and $\epsilon^{\prime \prime}$ (gray lines), including a power law in $\epsilon^{\prime \prime}$ for conductivity. The ranges of the symmetric stretching exponent $\beta$ of the HN functions for electrode polarization (EP), the cluster relaxation (CR), and the segmental relaxation $\left(\tau_{\alpha}\right)$ are given. (b) Relaxation map (Arrhenius plot) for all samples, including DMA results for the chain dynamics (big open symbols; see Figure 9c below). The thick straight and curved lines are Arrhenius and Vogel-Fulcher fits, respectively. $E_{\mathrm{a}}^{\text {app }}$ values for EP and the CR in the high- $T$ range (linear fits, thin black lines) are indicated. The Barton-Nakajima-Namikawa (BNN) correlation between the conductivity $\sigma_{0}$ and the characteristic frequency of EP shown in the inset proves their common origin. Open symbols for sample BIIR-BI are as-measured, before correction of the larger sample thickness (see the SI). (c) Relaxation strength $\Delta \epsilon_{\mathrm{CR}}$ of the cluster relaxation. The linear fits just guide the eye.

average, $\left.10^{\langle\log (\tau)\rangle}\right)$, and a symmetric stretching exponent $\beta$ describing the width of the $\tau$ distribution (the asymmetric stretching exponent $\gamma=1$ was fixed; see the SI). In the range of lower temperatures, we observe the segmental $\alpha$ process and a slower and higher-amplitude relaxation that we attribute to the ionic clusters (cluster relaxation, CR). The latter relaxation is absent in pure BIIR, and the former roughly corresponds to its segmental relaxation; see the relaxation map in Figure $3 \mathrm{~b}$, lower right. The relation between $\tau_{\mathrm{CR}}$ and the sticker lifetime $\tau_{\mathrm{st}}^{*}$ turns out to be nontrivial and will be discussed in later sections.

The $\alpha$ process in $\mathrm{PIB}^{62,63}$ as well as in halogenated butyl rubber $^{64-66}$ was studied previously, and it roughly matches with our results on pure BIIR as far as the relaxation strength, the time scale $\left(\tau_{\alpha}\right)$ as well as the stretching exponent $\beta$ are concerned. Pure PIB has a very low dipole moment, resulting in a rather low relaxation strength, with $\epsilon^{\prime \prime}$ only reaching a value of about 0.003 at maximum loss. In halogenated butyl rubber such as BIIR, the response is about 10 -fold due to the large local dipole moment, yet $\tau_{\alpha}$ and $\beta$ still reflect the segmental relaxation of the bulk PIB matrix due to the $\mathrm{C}-\mathrm{Br}$ dipole acting as a dilute probe. The " $\alpha$ process" detected in the ionomers is shifted toward somewhat lower $\tau_{\alpha}$ as compared to pure BIIR. We remind that this relaxation (with its comparably large $\Delta \epsilon$ of around $0.03-0.05$, which is on average only somewhat lower than that of pure BIIR) is associated with the polar sidegroups that are mostly associated with the ionic clusters. We may therefore assume that it is in this case not representative of the BIIR bulk but of the material close to the clusters. Further, it is possible that it is subject to a bias related to neglecting a faster $\beta$ process that can be observed and fitted in pure BIIR but not in the ionomers due to experimental noise. The deviation is thus not very significant. The rheological data discussed below will provide clear proof that $\tau_{\alpha}$ of the PIB matrix is actually unchanged.

The CRs dominate the central part relaxation map in Figure $3 \mathrm{~b}$, and their temperature dependencies fit well the VogelFulcher relations with Vogel temperatures that are all within about $20 \mathrm{~K}$ of $T_{\mathrm{V}}$ for $\tau_{\alpha}$ of pure BIIR (124 $\mathrm{K}$ in our case). The only exception is the sample BIIR+BI, which was from an older batch and appears to have a somewhat lower $T_{\mathrm{g}}$ and $T_{\mathrm{V}}$, possibly due to some residual solvent or water. On the hightemperature side (left in the Arrhenius plot), the CRs feature steeper slopes and thus higher $E_{\mathrm{a}}^{\text {app }}$ than $\tau_{\alpha}$ of pure BIIR (consistently, BIIR+BI is again an outlier). These relations between $\tau_{\mathrm{CR}}(T)$ and $\tau_{\alpha}(T)$ provide a confirmation for the validity of eq 1 , assuming for the moment a direct relation between $\tau_{\mathrm{CR}}$ and $\tau_{\mathrm{st}}^{*}$. In other words, if the matrix freezes upon passing the glass transition, the cluster dynamics will also be arrested. The related relaxation strength $\Delta \epsilon_{\mathrm{CR}}$ (see Figure 3c) may be taken as a measure of the angular amplitude of the cluster relaxation. It increases only weakly with $T$ but shows a prominent increase from about 0.27 to 0.55 on going from BIIR+MI to BIIR+NI. The comparably small $\Delta \epsilon$ suggests that the $\mathrm{CR}$ is not associated with dipole reorientations of contact ion pairs, in agreement with recent BDS and light scattering studies of related ionic liquids. ${ }^{67}$

At higher temperatures, the $\mathrm{CR}$ is observed at the highfrequency end of the spectrum, preceded by a rather weak feature $\left(\mathrm{CR}^{\prime}\right)$ that we also attribute to the ionic clusters, which may be related to inhomogeneities or impurities. A large upturn in $\epsilon^{\prime \prime}$ due to conductivity follows toward the lowest frequencies. A look at $\epsilon^{\prime}$ and the analysis of its derivative (see the SI) reveal another very prominent relaxation hidden below the conductivity upturn, which we attribute to electrode polarization (EP). Notably, $\tau_{\mathrm{EP}}$ and the conductivity are directly linked, exhibiting the very same activation energies, as is apparent from the Barton-Nakajima-Namikawa (BNN) correlation $^{68,69}$ shown in the inset of Figure $3 \mathrm{~b}$. See Figure S2 for an Arrhenius plot of the conductivity $\sigma_{0}$.

At this point, we anticipate relevant DMA results on the cluster-related chain relaxation times that are also shown in Figure $3 b$. These exhibit the same order and similar $E_{a}^{a p p}$ across the sample series as EP, which suggests that all of these processes reflect the motion of charge-carrying imidazole sidegroups through the BIIR matrix. This is at first glance 
surprising, considering that the more freely mobile $\mathrm{Br}^{-}$ counterion should dominate the conductivity. However, a close dynamic correlation of both ions, for example, through a carrier mechanism through the unpolar matrix, can be envisioned and has in fact been observed in moleculardynamics simulations of polymeric ionic liquids. ${ }^{70}$ A more detailed discussion of the relationship of the BDS-based CR and the NMR- and DMA-based potential counterparts will be provided below.

III.III. Principle of NMR Experiments. We now discuss the NMR results, which reflect the molecular dynamics of individual segments, sticky groups, and whole chain segments, thus providing a link to the mechanical properties. All NMR methods used herein rely on measuring the dipole-dipole couplings (DDCs) between abundant protons, which at fixed distance depend on the spin pair orientation via $\omega_{\mathrm{D}}(t)=$ $D_{\text {stat }} P_{2}(\cos \theta(t))$, where $D_{\text {stat }}$ is the static-limit coupling constant, $P_{2}$ is the second Legendre polynomial, and $\theta$ is the segmental orientation with respect to the external magnetic field $B_{0}$. When segments start fluctuating ( $\alpha$ process) with correlation time $\tau \ll 1 / D_{\text {stat }} \sim 10 \mu$ s but subject to the connectivity constraint, the measurable time-averaged coupling is reduced to a very weak time-averaged residual coupling (RDCC), $D_{\text {res }}=\left\langle\omega_{\mathrm{D}}\right\rangle$. All measured couplings are generally second-moment-type quantities, that is, they represent an average of multiple couplings. ${ }^{71}$

The observable $D_{\text {res }}$ is commonly related to a local dynamic order parameter

$$
S=\left\langle P_{2}\left(\cos \vartheta_{t}\right)\right\rangle_{t}=D_{\text {res }} / D_{\text {eff }}
$$

where $\vartheta_{t}$ is now the fluctuating segmental orientation with respect to the symmetry axis of motion. It characterizes the degree of (transient) anisotropy of the motion. $D_{\text {eff }}$ is a suitably defined preaveraged static-limit DDC constant (in $\mathrm{rad} / \mathrm{s}$ ) describing a rigid system but corrected for very fast motions inside Kuhn segments (on a ps time scale). Since time averaging is implicit to the experiment performed on a given time scale $t_{\exp }$ in the time domain, the measured $D_{\text {res }}$ will in general be temperature-dependent. Only for permanent networks at sufficiently high $T$ will $S \propto D_{\text {res }}$ be $T$-independent, and $S=3 / 5 N$ is then related to the cross-link density $1 / N(N$ being the number of Kuhn segments between constraints).

The interpretation of ${ }^{1} \mathrm{H}$ NMR results can proceed either by assuming a quasi-static $S \propto D_{\text {res }}$ and interpreting its $T$ dependence (thus neglecting transverse-relaxation effects arising from the presence of motion on the experimental time scale) or by explicit consideration of the time dependence of $P_{2}(\cos \vartheta(t))$. In the former case, time-temperature superposition (TTS) provides a link to the OACF $C(t)$ noted in the Introduction because $C\left(t_{\exp }, T\right) \sim S^{2}(T)$. This is the strategy that we have applied in our previous study of ionomeric rubbers. ${ }^{14}$ In the latter case, one attempts to deduce the shape of $C(t)$ from the data. We have done this previously on the example of linear entangled melts, ${ }^{43}$ and below, we will compare both approaches on the given class of materials.

III.IV. Segmental Dynamic Heterogeneity. Ionomers are known to feature strong dynamic heterogeneity in a sense that polymer segments in proximity of the ionic clusters may exhibit a shifted glass-transition temperature. ${ }^{11-13}$ These dynamically distinct regions can even act as a reinforcing filler of the elastomer matrix ${ }^{14}$ and thus be responsible for significant changes in the material's mechanical properties. Following our previous work, ${ }^{14}$ time-domain NMR can be used to detect the presence of immobilized segments with strongly increased $\tau_{\alpha}$.

Similar to the case of semicrystalline or block copolymers with large dynamic contrast, the simple ${ }^{1} \mathrm{H}$ time-domain signal decay (free-induction decay, FID) can be analyzed in terms of multiple, dynamically distinct components ${ }^{72}$ characterized by either $D_{\text {stat }}$ or specific $D_{\text {res }}$ values. Results for our samples along these lines are collected in the SI; see Figure S3. They simply demonstrate the absence of any significant amount of strongly immobilized polymeric components. Considering our detection limit of a few $\%$ of the total proton signal, the ionic groups either are too sparse, forming only comparably smaller multiplets that would only lead to immobilization on the scale of the persistence length, ${ }^{73}$ or have a high internal mobility-as actually indicated by BDS.

III.V. Quasi-Static Analysis of ${ }^{1} \mathrm{H}$ DQ NMR Data. To characterize the degree of motional anisotropy, ${ }^{1} \mathrm{H}$ DQ NMR experiments were performed under static and, to gain better resolution to distinguish imidazolium signals, MAS conditions. In the static case, a DQ pulse sequence based on the one developed by Baum and Pines ${ }^{74}$ was utilized, ${ }^{51}$ whereas in the case of the high-resolution measurements under MAS, the DQ recoupling pulse sequence BaBa-xy $16^{75}$ was employed.

These methods rely on coherent spin evolution under the action of dipolar coupling during a pulse sequence duration $\tau_{\mathrm{DQ}}$, and the signal functions depend on the time-integrated dipolar coupling, that is, the phase $\phi=k \int_{0}^{\tau_{\mathrm{DQ}}} \omega_{\mathrm{D}}(t) \mathrm{d} t$ ( $k$ being a pulse-sequence-dependent scaling factor). ${ }^{71}$ Either for the total signal at low resolution (dominated by the rubber matrix) or for the resolved resonances under MAS, we record depending on the phase cycle either a DQ buildup function

$$
I_{\mathrm{DQ}}\left(\tau_{\mathrm{DQ}}\right)=\left\langle\sin ^{2} \phi\right\rangle
$$

or a reference decay function $I_{\text {ref }}=\left\langle\cos ^{2} \phi\right\rangle$. The sum of the two signal functions gives a fully dipolar refocused relaxationonly signal

$$
I_{\Sigma \mathrm{MQ}}\left(\tau_{\mathrm{DQ}}\right)=\left\langle\sin ^{2} \phi\right\rangle+\left\langle\cos ^{2} \phi\right\rangle
$$

(This "sum multiple-quantum" signal also involves refocused higher-order coherences, which is why we often refer to this group of methods as "MQ NMR.")

Finally, to remove relaxation effects of fast motions occurring at times $t<\tau_{\mathrm{DQ}}$, a normalization of the DQ signal is done via the point-by-point division, $I_{\mathrm{nDQ}}=I_{\mathrm{DQ}} / I_{\Sigma \mathrm{MQ}}$. This procedure is only successful when $C(t)$ (see Figure $1 \mathrm{~b}$ ) does not decay appreciably on the $\tau_{\mathrm{DQ}}$ time scale, then leading to $I_{\mathrm{nDQ}}\left(\tau_{\mathrm{DQ}}\right)$ reaching a value of 0.5 in the long-time limit. The successful normalization is demonstrated on a sample nDQ buildup curve plotted in Figure 4a. $D_{\text {res }}$ is then determined by a fit using an empirical, universal buildup function valid for rubbers with protons close to the main chain ${ }^{76}$

$$
\begin{aligned}
I_{\mathrm{nDQ}}\left(\tau_{\mathrm{DQ}}\right)= & 0.5\left(1-\exp \left\{-\left(0.378 D_{\mathrm{res}} \tau_{\mathrm{DQ}}\right)^{1.5}\right\}\right. \\
& \left.\times \cos \left(0.583 D_{\mathrm{res}} \tau_{\mathrm{DQ}}\right)\right)
\end{aligned}
$$

For the case of $\mathrm{BaBa}-\mathrm{xy} 16$, the two prefactors of $D_{\text {res }}$ need to be multiplied by $0.78 .^{75}$ Deviations from this function shape can arise from RDCC distributions, for which we have recently recommended using a log-normal probability density. ${ }^{77}$ The data in Figure 4a demonstrate a good fit using eq 7 as the Kernel function in a numerically calculated distribution integral. We can also see that the $\log -$ normal $D_{\text {res }}$ distribution 

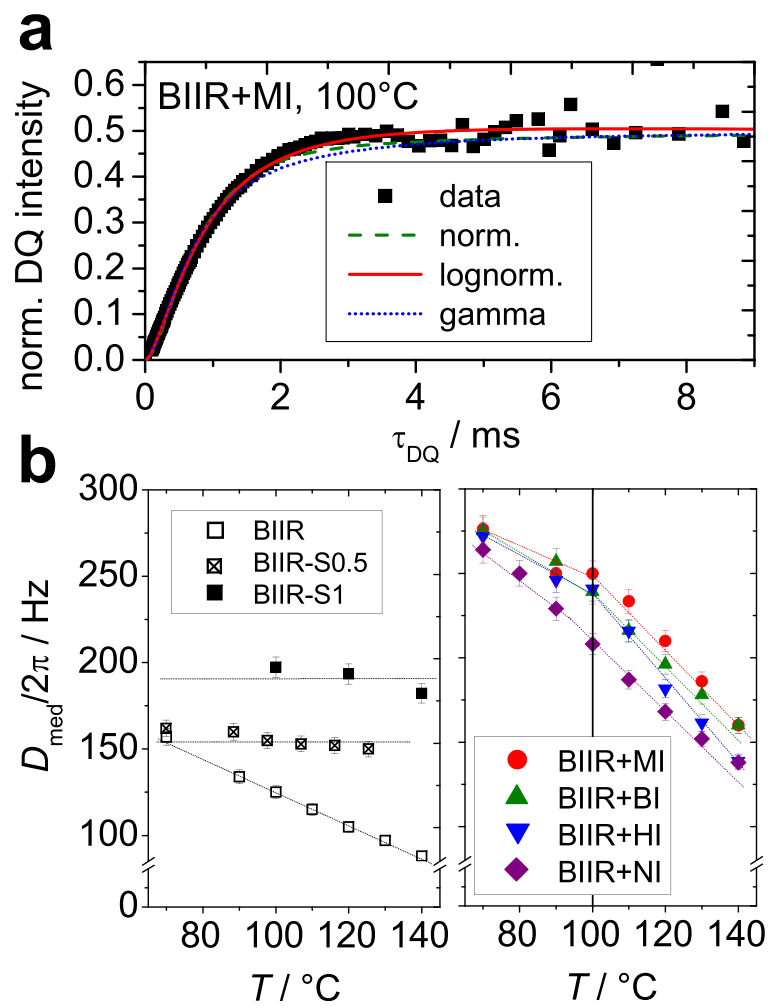

Figure 4. (a) Normalized DQ buildup curve, $I_{\mathrm{nDQ}}$, measured under static conditions and fitted to eq 7 further assuming different $D_{\text {res }}$ distributions, and (b) median RDCCs of a log-normal distribution, $D_{\text {med }} / 2 \pi$, as a function of temperature measured for nonionic samples (left panel) and the ionic BIIR networks (right panel).

provides better fits than the normal or $\gamma$ distributions used so far. ${ }^{77}$ In the following, we discuss the median RDCCs, $D_{\text {med }}=$ $\mathrm{e}^{\left\langle\ln D_{\mathrm{res}}\right\rangle}$. The apparent logarithmic distribution widths are to a good part due to shape changes of $I_{\mathrm{nDQ}}\left(\tau_{\mathrm{DQ}}\right)$ arising from intermediate-motional effects, that is, nonconstant $C(t)$, as discussed in the SI.

III.VI. Network Structure. The $D_{\text {med }}$ data in Figure $4 \mathrm{~b}$ characterizing the average constraint density as a function of temperature could only be obtained above $70{ }^{\circ} \mathrm{C}$, where the normalization procedure is successful (i.e., where the quasistatic approximation works). As to the nonionic samples (left panel), the results for BIIR-S0.5 and -S1 being permanent networks hardly change with temperature, as expected, whereas pristine BIIR exhibits a significant reduction of $D_{\text {med }}$ with temperature as a result of regime II (constrained Rouse) dynamics. Notably, at $70{ }^{\circ} \mathrm{C}$, BIIR exhibits nearly the same value of $D_{\text {med }}$ as BIIR-S0.5, which means that its cross-link spacings are much larger than the entanglement distance.

The ionic networks (right panel of Figure $4 \mathrm{~b}$ ) show even stronger thermal evolution than BIIR, which implies that apart from the segmental motion, there is an additional mechanism averaging $D_{\text {med }}$, which is of course associated with the sticky dynamics. ${ }^{19,31}$ Also, one can immediately see that all ionic networks have the same initial apparent cross-link density at $70{ }^{\circ} \mathrm{C}$, which is by about a factor of 2 larger than the entanglement level observed for BIIR and BIIR-S0.5. This factor is expected, as discussed below. At $70{ }^{\circ} \mathrm{C}$, the dynamics of ionic links is thus too slow and does not lead to the appreciable chain relaxation. In our recent contribution, ${ }^{4}$ we have only studied temperatures starting at $100{ }^{\circ} \mathrm{C}$ (marked by the vertical line), above which $D_{\text {med }}$ depends systematically on the alkyl side chain length and shows a strong decrease with $T$, arising from chain relaxation following increasingly frequent sticker detachment from the clusters.

III.VII. Anisotropy of Sticker Motion. To study structural and dynamic properties of AI groups serving as anchors binding polymer chains in a common ionic network, we resort to high-resolution DQ MAS experiments. Only above $100{ }^{\circ} \mathrm{C}$ we observed sufficiently resolved spectra at $10 \mathrm{kHz}$ spinning. The uniform line shapes seen in Figure 5, the absence of any significantly sharper peaks, and the DQ data discussed below provide good indications for the absence of a significant amount of free, isotropically mobile stickers persisting on the millisecond (ms) time scale. Even though this is expected for the given, strongly associative case, a fraction of mobile binary assemblies has for instance been postulated for cluster-forming acrylic acid moieties in more polar ionomers. ${ }^{27}$

The integral ratio of the single imidazole proton $\mathrm{H}_{1}$ to the other two is somewhat larger than 2, which is straightforwardly attributed to a residual contribution from the comparably very large aliphatic signal from the alkyl tail and the dominant BIIR backbone, resulting from the limitations arising from removing the latter via baseline correction. The integral ratio of $\mathrm{H}_{1}$ to the latter is, however, well in line with the stoichiometry of the sample, meaning that no signal, possibly related to fully rigid clusters (for which $10 \mathrm{kHz}$ MAS would not be sufficient for resolved peaks), is lost in a broad baseline. The rather narrow lines and further narrowing on heating suggest rather fast intracluster motion and an increase in motional rate and/or amplitude on heating.

For the BaBa-based DQMAS experiments, the spectrometer frequency was set close to the imidazolium signals in the spectral range of around $7-8 \mathrm{ppm}$ to avoid resonance-offset effects. For buildup analysis, we did not observe significant

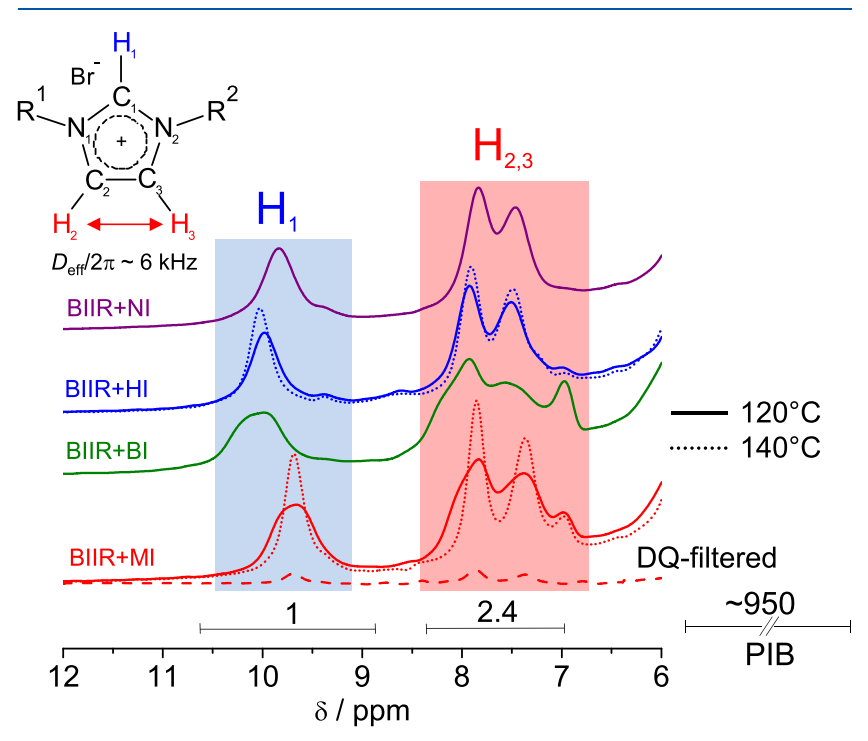

Figure 5. One-dimensional (1D) MAS spectra of the imidazolium signals in the ionic networks at $120^{\circ} \mathrm{C}$ (solid lines) and $140{ }^{\circ} \mathrm{C}$ (two samples only, dotted lines), separated vertically for clarity. The DQfiltered spectrum (dashed line) was acquired with $\tau_{\mathrm{DQ}}=2 \tau_{\mathrm{R}}=0.2 \mathrm{~ms}$ at $140{ }^{\circ} \mathrm{C}, \tau_{\mathrm{R}}$ being the rotor period. The integral ratios of the DQfiltered signals are roughly equal to the indicated integral ratios of the unfiltered signals (1:2.0). The intra-imidazole next-neighbor coupling indicated along the sketched chemical structure is based on bond lengths and angles taken from the literature. ${ }^{57,78,79}$ 
differences among the different imidazolium resonances (see Figure S5 in the SI). This is ultimately the result of the dipolar truncation effect and significant intermolecular couplings, ${ }^{71}$ and it shows that the imidazolium groups, despite being rather mobile, are well packed within the ionic clusters.

The $I_{\mathrm{nDQ}}$ data of the $\mathrm{H}_{2,3}$ signals of all of the ionic samples are compared in Figure 6. The left panel shows a comparison of all samples at $120^{\circ} \mathrm{C}$, for which we observe a slower buildup (lower $D_{\text {med }}$ ) upon increasing the length of the alkyl group in the alkylimidazolium moiety, corresponding to more isotropic dynamics. This trend matches the BDS observation of increasing $\Delta \epsilon_{\mathrm{CR}}$ (see Figure $3 \mathrm{c}$ ). $D_{\text {med }}$ also decreases upon increasing the temperature (right panel), which supports the finding of Figure 4, where we have also observed a drop of $D_{\text {med }}$ of the BIIR chains upon increasing $T$ and the alkyl tail length. Thus, we can directly confirm the important role of the alkyl group in reducing the strength of the ionic links. The buildup curves were again fitted, with results tabulated and compared to those for network chains at the same temperatures in Table 1.

The comparison is surprising considering that the intracluster residual couplings are only a factor of 4 (for BIIR+MI) larger or even about the same as for the network chains. Even taking into account that the strongest static-limit reference coupling for the imidazolium moieties ( $\sim 6 \mathrm{kHz}$; see Figure 5) is about a factor of 3 smaller than the one for the $\mathrm{CH}_{2}$ groups in the main chains, we can conclude that we have rather largeamplitude orientational mobility within the ionic clusters ${ }^{80}$ and thus very low order parameters $S$ (see eq 4 ), with values on the order of $S=0.13$ for BIIR+MI down to 0.02 for the other samples. As to the time scale, the relevant correlation time needs to be much shorter than the inverse coupling $(2 \pi \times 6$ $\mathrm{kHz})^{-1} \approx 25 \mu \mathrm{s}$; the data in Figure $3 \mathrm{~b}$ immediately confirms that this is the case for $\tau_{\mathrm{CR}}$ of all samples. At present, we cannot decide whether the observations are due to a larger timeaveraged fraction of free stickers (fast exchange on the sub-ms scale) or due to an actually more isotropic dynamics within the

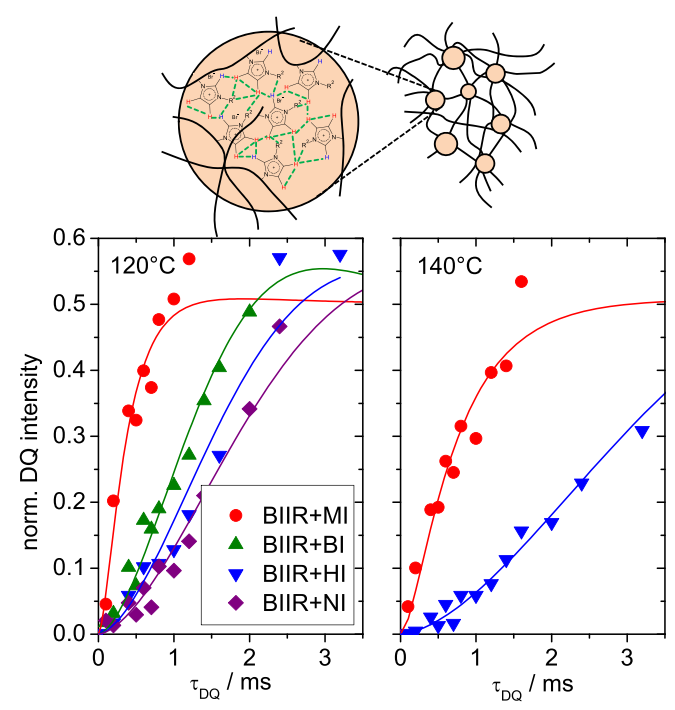

Figure 6. Normalized DQ buildup curves of the alkylimidazolium signals of all of the ionic samples at 120 and $140{ }^{\circ} \mathrm{C}$. The solid lines correspond to fits to eq 7, additionally assuming a log-normaldistributed $D_{\text {res }}$. The sketch at the top illustrates the multispin intraand intermolecular dipolar-coupling network within the ionic aggregates.
Table 1. Median RDCCs from ${ }^{1} \mathrm{H}$ DQ NMR Analysis of the Ionic Stickers As Compared to the Network Chains

\begin{tabular}{|c|c|c|c|c|c|c|}
\hline \multirow[b]{3}{*}{ sample } & \multicolumn{3}{|c|}{ ionic stickers } & \multicolumn{3}{|c|}{ network chains } \\
\hline & \multicolumn{2}{|c|}{$D_{\text {med }} / 2 \pi(\mathrm{Hz})$} & \multirow{2}{*}{$\frac{D_{\text {med }}^{120^{\circ} \mathrm{C}}}{D_{\text {med }}^{140{ }^{\circ} \mathrm{C}}}$} & \multicolumn{2}{|c|}{$D_{\text {med }} / 2 \pi(\mathrm{Hz})$} & \multirow{2}{*}{$\frac{D_{\text {med }}^{120^{\circ} \mathrm{C}}}{D_{\text {med }}^{140{ }^{\circ} \mathrm{C}}}$} \\
\hline & $120{ }^{\circ} \mathrm{C}$ & $140{ }^{\circ} \mathrm{C}$ & & $120^{\circ} \mathrm{C}$ & $140{ }^{\circ} \mathrm{C}$ & \\
\hline $\mathrm{BIIR}+\mathrm{MI}$ & 802 & 395 & 2.04 & 210 & 160 & 1.32 \\
\hline BIIR+BI & 268 & & & 196 & 160 & 1.22 \\
\hline $\mathrm{BIIR}+\mathrm{HI}$ & 206 & 106 & 1.96 & 182 & 139 & 1.32 \\
\hline $\mathrm{BIIR+NI}$ & 170 & & & 168 & 138 & 1.22 \\
\hline BIIR & & & & 105 & 88 & 1.19 \\
\hline
\end{tabular}

clusters but no significant exchange with a population of free stickers (we have already excluded the presence of a timestable free sticker fraction).

A last remark concerns the ratios of the $D_{\text {med }}$ values at 120 and $140{ }^{\circ} \mathrm{C}$; see also Table 1 . One can notice that the $D_{\text {med }}$ ratios of the ionic links are larger than the ones of the polymer backbones. This indicates a more prominent acceleration and isotropization of intracluster dynamics as compared to the chain dynamics. Further, there is no significant effect of the length of the alkyl group on the $D_{\text {med }}$ ratios (for both ionic links and backbone) at the given, large temperatures. This supports the notion that the chain-based relaxations are decoupled from details of the intracluster dynamics. This point will be taken up below.

III.VIII. Microscopic Network Chain Dynamics. The DQ NMR data can deliver even more quantitative information on the molecular dynamics than probed so far only qualitatively via the temperature dependence of $D_{\text {med }}$. We now try to assess the shape of the OACF; see Figure $1 \mathrm{~b}$. While one can in principle use the initial rise of $I_{\mathrm{nDQ}}\left(\tau_{\mathrm{DQ}}\right)$ in combination with TTS, as successfully demonstrated for entangled melt dynamics, ${ }^{43}$ this approach is subject to a noise penalty and critical for inhomogeneous systems ${ }^{53}$ and when the shift parameters are not well-defined.

We thus follow a recent analytical fitting approach presuming a certain shape of the OACF in the fitted realtime interval ranging from the minimal $(\sim 0.1 \mathrm{~ms})$ to a maximal (order of $1 \mathrm{~ms}$ ) $\tau_{\mathrm{DQ}}$. A power-law model is reasonable for dynamics in soft materials

$$
C(t) \propto \begin{cases}D_{\text {res }}^{2}\left(t_{0}\right) & \text { for }|t|<t_{0} \\ D_{\text {res }}^{2}\left(t_{0}\right)\left(t / t_{0}\right)^{-\kappa} & \text { for }|t| \geq t_{0}\end{cases}
$$

where $D_{\text {res }}\left(t_{0}\right)$ is the RDDC at $t_{0}$, which is set fixed at the time just below the first experimental point $\left(\tau_{\mathrm{DQmin}}\right)$, and $\kappa$ is the power-law exponent. The explicit fitting model is algebraically somewhat cumbersome and can be found in ref 53. It includes another exponential $T_{2}$ term to account for motions on time scales below $t_{0}$. The fit is stable when the DQ and MQ signals, eqs 5 and 6 , are fitted simultaneously. For cases where the correlation loss is not a power law (e.g., an exponential), the fit provides a force-fitted approximation in a suitably short fitting interval (i.e., the local slope in a log-log plot such as in Figure 1b). This is why the choice of the fitting interval is a compromise between the force-fitted approximation (which becomes potentially worse for longer intervals) and the stability and precision of the fit (which suffers for short intervals). A sample fit is shown in Figure $7 \mathrm{a}$, and further details as well as a discussion of an analogous analysis of the 
MAS NMR results for the imidazolium groups are deferred to the SI.

In Figure $7 \mathrm{~b}, \mathrm{c}$, the $D_{\text {res }}\left(t_{0}\right)$ and $\kappa$ values obtained for the rubber matrix are presented as a function of temperature. It can be seen that both the $D_{\text {res }}\left(t_{0}\right)$ and $\kappa$ values of all of the samples start around $800 \mathrm{~Hz}$ and $0.6-0.7$ at $30{ }^{\circ} \mathrm{C}$, respectively, and first quickly decrease with temperature and then level off. This corresponds to the transition from Rouse regime I to either the "Leibler plateau" at $\tau_{\mathrm{x}}$ (see Figure $1 \mathrm{~b}$ ) for the network samples or the tube-constrained regime II for linear BIIR. ${ }^{43}$ The theoretical versus experimental high-MW limiting slopes for the latter are 0.25 versus $0.29 .{ }^{45,48}$ Even lower values indicate more constrained dynamics arising from cross-links.

As the cross-link density increases on the order of BIIR, BIIR-S, and BIIR+AI, the transition of the $\kappa$ values shifts to lower temperatures, as expected, since the network chains start feeling the constraints earlier. Beyond $100{ }^{\circ} \mathrm{C}, D_{\text {res }}\left(t_{0}\right)$ delivers similar trends as already seen in the context of the data in Figure $4 \mathrm{~b}$, whereas the $\kappa$ values in the nonionic samples either stay constant or continue to decrease (BIIR-S) beyond $100{ }^{\circ} \mathrm{C}$, yet more slowly, indicating even more constrained dynamics. The nonzero $\kappa$ for BIIR-S can be the result of relaxation of defects or chain motions within the tube. ${ }^{81}$

In contrast to the nonionic samples, the ionic networks exhibit an increase of $\kappa$ values, again reaching the exponent of a delayed regime II of 0.29 at the highest studied temperatures. This marks the end of the Leibler plateau due to the opening of the sticky bonds. The idealized sticky-reptation prediction (Figure $1 b$ ) would suggest a short sticky Rouse regime with $\kappa$ exceeding 0.5. This is not observed, and we attribute this to crossover effects and a wide distribution of sticky correlation times, as confirmed by BDS and also DMA below. It is also

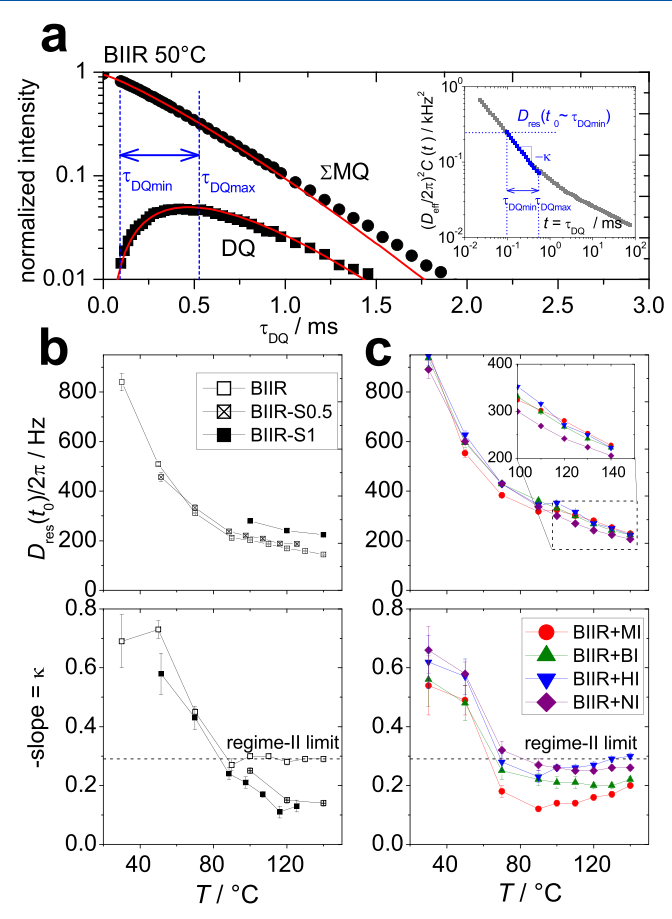

Figure 7. (a) Power-law-based fit $^{53}$ (red solid lines) on the example of BIIR at $50{ }^{\circ} \mathrm{C}$. The inset shows a part of a piecewise-constructed $C(t)$ in $\log -\log$ representation, approximated linearly within the fitting interval between $\tau_{\mathrm{DQ} \min }$ and $\tau_{\mathrm{DQ} \max } \cdot(\mathrm{b}, \mathrm{c})$ Temperature dependence of the extracted $D_{\text {res }}\left(t_{0}\right)$ and $\kappa$ values (see eq 8 ) of nonionic (b) and ionic (c) samples. seen that $\kappa$ is on average larger for longer alkyl groups of the alkylimidazolium moieties, which indicates an earlier transition to sticky reptation and thus an overall shorter Leibler plateau.

We now use the obtained $D_{\text {res }}\left(t_{0}\right)$ and $\kappa$ values, providing piecewise linear parts of $C(t)$ in a log-log plot, to construct master curves by TTS. ${ }^{34,35}$ An example is shown in the inset of Figure $7 \mathrm{a}$, and the whole procedure is illustrated in Figure S6a in the SI. We have decided to use $T_{\text {ref }}=50^{\circ} \mathrm{C}$, which is well in the Rouse regime where the $C(t)$ for all samples should coincide. We can now go ahead and determine characteristic transition times, as indicated in the same figure. See Figure S6b for details on the derivative analysis used to estimate the entanglement time $\left(\tau_{\mathrm{e}, \text { exp }}\right)$ on the example of BIIR at $T_{\text {ref }}=50$ ${ }^{\circ} \mathrm{C}$. The result of $0.77 \mathrm{~ms}$ is in good agreement with a theoretical estimate of $0.46 \mathrm{~ms}$ based on the known friction coefficient; see the SI.

$C(t)$ master curves for all samples are compared in Figure 8a. All ionic samples indeed coincide with pure BIIR at short times in the Rouse regime, which proves the essentially unchanged $\alpha$ relaxation of the PIB matrix. As expected, the ionic samples exhibit an enhanced quasi-plateau and a delayed regime II, which sets in beyond $\tau_{\mathrm{e}}$ or its sticky equivalent $\tau_{\mathrm{e}}^{*}$, with BIIR+MI showing the largest delay. As discussed above in the context of the $\kappa$ values, we do not observe a clear Leibler plateau $(\kappa=0)$ and a short steeper sticky-Rouse regime, but this is not surprising considering the correlation time distribution and the corresponding transitional effects. We therefore list in Table 2 the $\tau_{\mathrm{e}}^{*}$ times at which $C(t)$ reaches the bare entanglement level $C_{\mathrm{e}}$. According to the sticky-reptation model (see Figure 1), ${ }^{19,21} \tau_{\mathrm{e}}^{*}=\tau_{\mathrm{st}}^{*}\left(N_{\mathrm{e}} / N_{\mathrm{st}}\right)^{2}$. Since the ratio $N_{\mathrm{e}} / N_{\text {st }}$ is close to unity in our case, $\tau_{\mathrm{e}}^{*}$ is on the same order as $\tau_{\mathrm{st}}^{*}$. For the amplitude of $C(t)$ at $\tau_{\mathrm{x}}$ as compared to $C_{\mathrm{e}}$, we expect a related factor of $\left(1+N_{\mathrm{e}} / N_{\mathrm{st}}\right)^{2} \approx 4 .^{88}$ This is robustly observed (see again Figure $8 \mathrm{a}$ ), to be confirmed by DMA. This aspect and the potential issues with master curve construction (see the gray boxes) will be taken up below.*

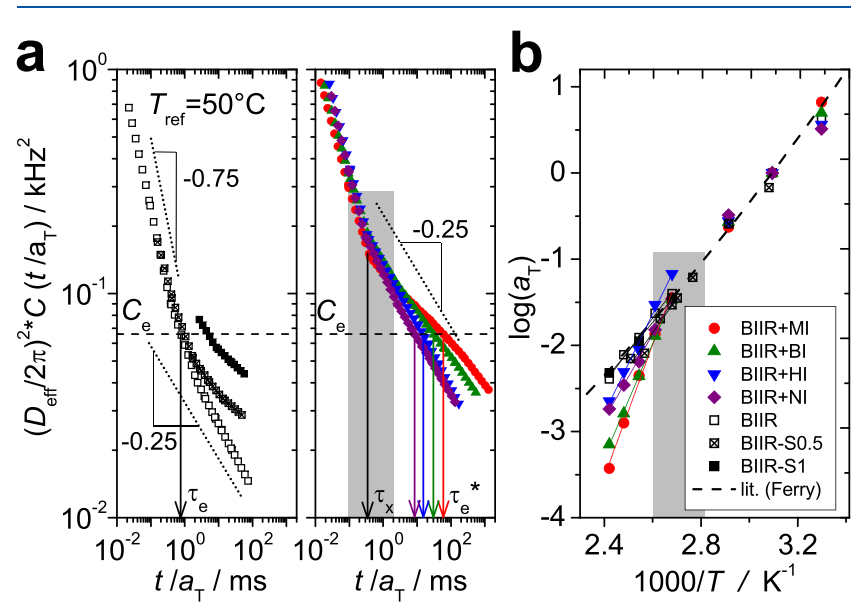

Figure 8. (a) Apparent $C(t)$ master curves of the nonionic (left) and ionic (right) samples reduced to $T_{\text {ref }}=50{ }^{\circ} \mathrm{C}$. The characteristic relaxation times are marked with arrows, and relevant power-law exponents are indicated. The dashed line indicates the anisotropy level defined only by entanglements $\left(C_{\mathrm{e}}=C\left(\tau_{\mathrm{e}}\right)=C\left(\tau_{\mathrm{e}}^{*}\right)\right)$. (b) Horizontal shift factors with solid lines corresponding to Arrhenius fits. The gray shaded boxes highlight the experimental time window in (a) where the shifting procedure transitions from being governed by the segmental to the sticky process, corresponding to an uncertainty at which temperature this transition occurs in (b). 
Table 2. Apparent Sticky Entanglement Times (in s) and Activation Energies ${ }^{a}$ (in $\mathrm{kJ} / \mathrm{mol}$ ) Taken from DQ NMR and DMA Master Curves and Shift Factors, Respectively

\begin{tabular}{|c|c|c|c|c|c|c|}
\hline \multirow[b]{2}{*}{ sample } & \multicolumn{3}{|c|}{$\operatorname{DQ} \operatorname{NMR}\left(T_{\text {ref }}=50{ }^{\circ} \mathrm{C}\right)$} & \multicolumn{3}{|c|}{$\operatorname{DMA}\left(T_{\text {ref }}=20{ }^{\circ} \mathrm{C}\right)$} \\
\hline & $\tau_{\mathrm{e}}^{(*)}$ & $\tau_{\mathrm{e}}^{*}\left(20^{\circ} \mathrm{C}\right)$ & $E_{\mathrm{a}}^{\mathrm{app}}$ & $\tau_{\mathrm{e}}^{*}$ & $E_{\mathrm{a}}^{\mathrm{app}}$ & $E_{\mathrm{a}}^{\text {st }}$ \\
\hline $\mathrm{BIIR}+\mathrm{MI}$ & $5.9 \times 10^{-2}$ & 1.01 & 147 & $>81.9$ & 113 & 52 \\
\hline $\mathrm{BIIR}+\mathrm{BI}$ & $3 \times 10^{-2}$ & 0.51 & 126 & 10.1 & 97 & 36 \\
\hline BIIR+HI & $1.5 \times 10^{-2}$ & 0.26 & 111 & 2.4 & 83 & 22 \\
\hline $\mathrm{BIIR+NI}$ & $8.5 \times 10^{-3}$ & 0.15 & 95 & 0.5 & 88 & 27 \\
\hline BIIR & $7.7 \times 10^{-4}$ & & & & & \\
\hline BIIR-S0.5 & $9.4 \times 10^{-4}$ & & 75 & & 61 & \\
\hline BIIR-S1 & & & 64 & & & \\
\hline BIIR lit./th. & $4.6 \times 10^{-443,54}$ & & & & & $61^{34}$ \\
\hline
\end{tabular}

${ }^{a}$ Fitted in a $T$ range around $120{ }^{\circ} \mathrm{C} ; E_{\mathrm{a}}^{\mathrm{st}}=E_{\mathrm{a}}^{\mathrm{app}}-E_{\mathrm{a}}^{\alpha}$ with $E_{\mathrm{a}}^{\alpha}\left(120{ }^{\circ} \mathrm{C}\right)=61 \mathrm{~kJ} / \mathrm{mol}$.
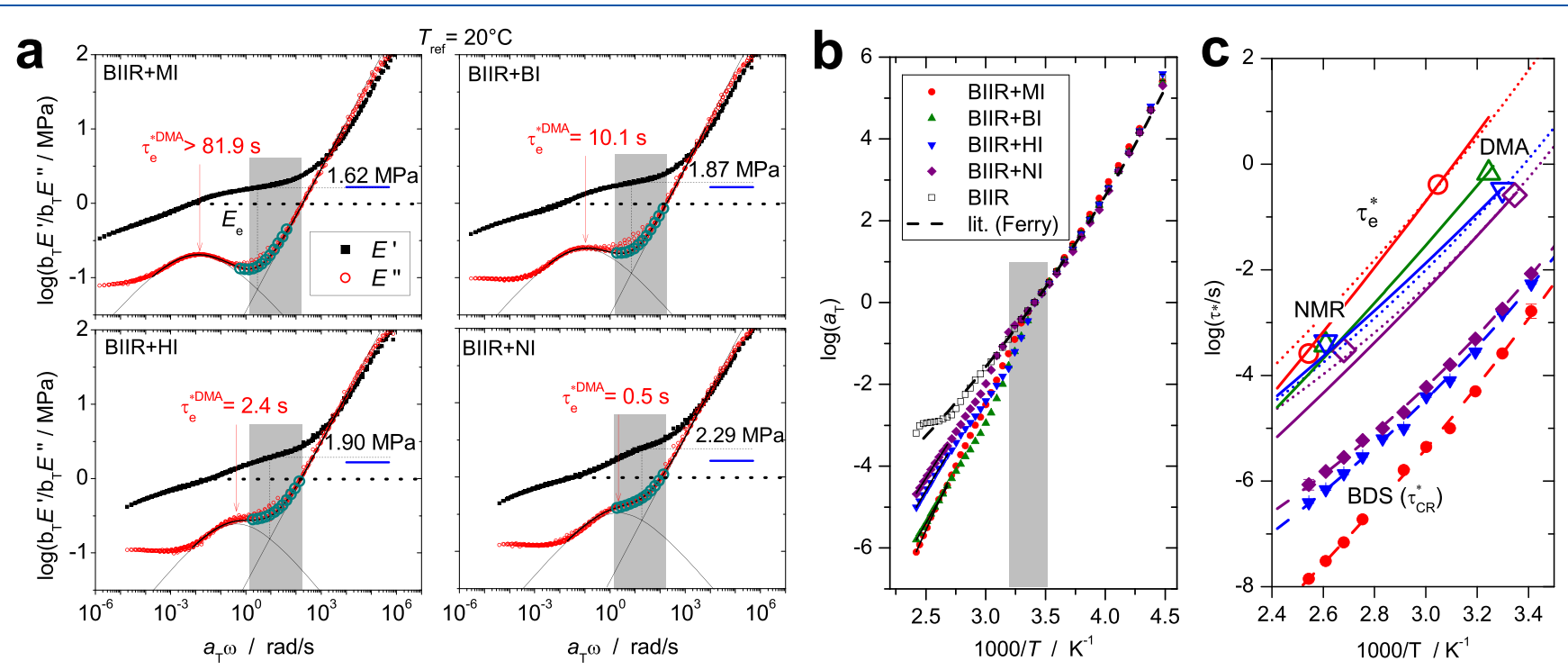

Figure 9. (a) Apparent $E^{\prime}$ and $E^{\prime \prime}$ master curves $\left(T_{\text {ref }}=20^{\circ} \mathrm{C}\right)$ for ionic samples. The thick solid lines are fits to an $\mathrm{HN}$ function combined with a power law for Rouse dynamics, shown separately as thin solid lines. The thick dotted and blue solid horizontal lines indicate the plateau modulus $\left(E_{\mathrm{e}}\right)$ of BIIR and the predicted higher-frequency plateau $\left(E_{\mathrm{N}}\right)$, and the thin dotted lines mark the stated actual values of the latter. The large open circles in $E^{\prime \prime}$ are from a single isothermal frequency sweep as explained in the text. (b) Horizontal shift factors including fits for $T$ between 20 and $140{ }^{\circ} \mathrm{C}$ considering the bulk VF/Williams-Landel-Ferry (WLF) relation and an additional activation barrier $E_{\mathrm{a}}^{\text {st }}$ (solid lines). The gray shaded boxes highlight the experimental time window at $T_{\text {ref }}=20^{\circ} \mathrm{C}$ in (a) where the shifting procedure transitions from being governed by the segmental to the sticky process, corresponding to an uncertainty at which temperature in (b) one chooses to switch from matching the high- vs the lowfrequency branch upon shifting. (c) Direct comparison of sticky entanglement times (see Figure 1) from DMA and NMR as measured in real frequency and time, respectively (open symbols). The solid lines are the shifted fits from (b), and the dotted lines are shifted VF fits to $\tau_{\mathrm{CR}}$ from BDS, the latter being also plotted as dashed lines and solid symbols, respectively.

The most relevant piece of information is the temperature dependence of the horizontal shift factors $\left(\log a_{\mathrm{T}}\right)$ as plotted in Figure $8 \mathrm{~b}$. We notice that the shift factors of the nonionic samples agree with the known WLF relation for butyl rubber. ${ }^{34}$ Arrhenius fits to the upper end of the temperature interval provide apparent activation energies as listed in Table 2. The overestimation on an average level of $15 \%$ as compared to the literature value of $61 \mathrm{~kJ} / \mathrm{mol}$ for $E_{\mathrm{a}}^{\mathrm{app}}=E_{\mathrm{a}}^{\alpha}$ of the nonionic samples is attributed to systematic errors in the afterall rather crude shifting procedure (note again that we shift force-fitted linear approximations to the real $\log C(\log t)$ for good overlap at the edges of the fitted time interval). Also, the need for a vertical shift (arising, e.g., from a $T$-dependent entanglement spacing) cannot be fully excluded, but its consideration is beyond the present scope. The higher $E_{\mathrm{a}}^{\text {app }}$ of the ionic samples is thus subject to a similar uncertainty, but the systematic decrease on going from BIIR+MI to BIIR+NI is a robust result.
III.IX. Mechanical Properties and Discussion of Apparent Master Curves. The DMA data taken in an angular frequency range of about $2-200 \mathrm{rad} / \mathrm{s}$ were also shifted to obtain apparent master curves, as shown in Figure 9a. As a reference temperature, we used $20^{\circ} \mathrm{C}$, at and below which data from all samples start to coincide on the highfrequency edge, corresponding to the Rouse regime. At any higher temperature, sticky dynamics controls the response of all samples.

For all samples, we can observe an indication for a Leibler plateau in $E^{\prime}$ at frequencies below the Rouse turnup. The level of this plateau $\left(E_{\mathrm{N}}\right)$ is defined by both stickers and entanglements. Assuming additivity, it can be estimated on the basis of the phantom model ${ }^{35}$

$$
E_{\mathrm{N}}=3 \rho R T\left(\frac{1}{M_{\mathrm{e}}}+\frac{1}{M_{\mathrm{st}}} \frac{f-2}{f} \frac{N_{\mathrm{br}}}{\mathrm{AN}}\right) \approx 1.7 \mathrm{MPa}
$$


where $\rho$ is the density of BIIR. We consider here the correction due to the predicted fraction of bridging chains, $N_{\mathrm{br}} / \mathrm{AN}$ (eq 3 ); the functionality of the cross-links (=clusters) also includes the following correction: $f=2 \mathrm{AN} N_{\mathrm{br}} / \mathrm{AN}=2 N_{\mathrm{br}}$ (each sticker involved in bridges has two chains attached). As indicated in Figure 9a, the theoretical prediction agrees very well with the experimental data for BIIR+MI, verifying that most of the stickers are in the closed state. The increasing deviations observed for the other samples can straightforwardly be attributed to the increasing proximity of the rather short Leibler plateau to the Rouse turnup.

The shift factors $a_{\mathrm{T}}$ plotted in Figure $9 \mathrm{~b}$ all coincide perfectly for $T$ below $20{ }^{\circ} \mathrm{C}$ in the Rouse regime and corroborate again a negligible change in $\tau_{\alpha}$ of the matrix, whereas above this temperature, they are dominated by the sticky dynamics. We thus have a larger $T$ range as compared to NMR (Figure 8b), reducing potential transitional effects of the TTS. We have analyzed $a_{\mathrm{T}}$ in the "sticky" range by fits based upon the bulk VF/WLF dependence and an additional activation barrier $E_{a}^{\text {st }}$, which is the rationale behind eq $1 .^{31,38}$ The corresponding $E_{\mathrm{a}}^{\mathrm{app}}(T)=E_{\mathrm{a}}^{\alpha}(T)+E_{\mathrm{a}}^{\text {st }}$ representing the slope at $T=120{ }^{\circ} \mathrm{C}$ (linearized VF, for a direct comparison with NMR and BDS results) are listed in Table 2. We notice that the $E_{\mathrm{a}}^{\text {app }}$ from NMR are systematically higher for all samples, which is attributed to larger uncertainties related to the master curve construction as discussed above. On the other hand, the DMA results agree within the uncertainties with the values for $\tau_{\mathrm{CR}}$ from BDS (see Figure $3 \mathrm{~b}$ ). This means that both appear to be subject to the same activation barrier.

Focusing now on the determination of the sticker lifetime, a clear indication for a maximum in $E^{\prime \prime}$ is observed for all samples although it is partially masked by the lack of overlap (indicating thermorheological complexity) in the frequency range above it and by the onset of the Rouse turnup. To locate the maximum in each case, we have fitted the $E^{\prime \prime}$ master curves (fitting $\log E^{\prime \prime}$ ) to a combination of the imaginary part of the Havriliak-Negami function (see the BDS part in the SI) and a power law, as also shown in Figure 9a. As to the $\mathrm{HN}$ parameters, the asymmetric stretching exponent $\gamma$ was always unity, whereas $\beta$ was around 0.4 in all cases, reflecting a distribution of the sticker lifetimes.

According to the model calculations in ref 21 , we expect the $E^{\prime \prime}$ maximum to be located at a lower frequency than $1 / \tau_{\mathrm{st}}^{*}$, and we associate it here with $1 / \tau_{\mathrm{e}}^{*}$. Due to $N_{\mathrm{st}} \approx N_{\mathrm{e}}$, both times are anyways very similar in our case (see also Figure 1a). We thus take the inverse frequency at the maximum location to be a good estimate of $\tau_{\mathrm{e}}^{*}$; see also Table 2 . The trend across the sample series reflects the same order as the NMR results, but the comparison of the numerical values requires some more discussion. It is important to stress that it is a priori expected that both methods probe the same type of chain relaxations (see Figure 1), at least at times/frequencies before the onset of terminal flow, where motions of whole clusters/micelles may become relevant. ${ }^{29}$ Note that also for NMR, a chain only relaxes (i.e., its OACF decays) if a sticker does not return to its original cluster.

For a closer comparison, we need to obtain results for the same temperature. Therefore, we also list in Table 2 the NMRbased $\tau_{\mathrm{e}}^{*}$ for $20{ }^{\circ} \mathrm{C}$. Estimating this is a rather simple exercise since we can just use the shift factor of about 17 given by the $\mathrm{VF} / \mathrm{WLF}$ relation of the bulk rubber in this temperature range. Notably, the resulting values are all by about 1-2 decades smaller than the DMA-based ones, suggesting (incorrectly!) strong disagreement. This illustrates an actual failure of TTS and stresses that the discussed master curves are apparent, also referred to as "pseudomaster curves." ${ }^{38}$ They are obviously only valid for one specific temperature-which is not necessarily $T_{\text {ref }}$.

This requires some explanations, which are partially based upon recent treatments of the same issue..$^{38,82}$ The problem is simply that the time-scale separation between the sticky regime $\left(\tau_{\mathrm{st}}^{*}\right.$ or $\left.\tau_{\mathrm{e}}^{*}\right)$ and the Rouse regime as controlled by $\tau_{0}(T)$ is temperature-dependent according to eq $1: \log \left(\tau_{\mathrm{e}}^{*}(T) / \tau_{0}(T)\right)$ $\propto E_{\mathrm{a}}^{\mathrm{st}} / R T$. This necessarily means that the breadth of the apparent master curve in frequency or time is a function of absolute temperature. This "shift-transition temperature" is the one where, upon increasing $T$, one chooses to change from shifting with regard to the best overlap in the high-frequency Rouse region to the low-frequency end when sticky dynamics starts to prevail. The frequency sweeps corresponding to the last (highest) temperature shifted to match the Rouse turnup are marked by large open circles in Figure 9a. The rest of the isothermal sweeps follow sticky dynamics at least at the lowfrequency end, and this also explains the lack of overlap in the gray shaded regions for the first of these data sets toward higher frequency. For three of the four samples, the shifttransition temperature corresponds to $T_{\text {ref }}=20{ }^{\circ} \mathrm{C}$, where in the Arrhenius plot (Figure 9b) the slope starts to change.

We can now understand why the shift factors for BIIR+MI apparently deviate (being shifted upward) in the Arrhenius plot; Figure 9b. In this case, the sticky dynamics becomes visible only at higher temperatures because it is not yet observed in the limited frequency range at $20^{\circ} \mathrm{C}$ (compare the large open circles in (a) to the shaded box). The higher shifttransition temperature is thus the reason for the shifted $a_{\mathrm{T}}$ values for BIIR+MI and its somewhat too low $\tau_{\mathrm{e}}^{*}$ value in Table 2. Therefore, also the frequency range of the experiment is shown to be relevant for the appearance of the master curves.

With this in mind, we can finally understand the $50 \mathrm{~K}$ difference between the apparent onsets of the sticky dynamics as seen by NMR (Figure 8b) and DMA (Figure 9b). The NMR time window covers much shorter times, namely, 0.1 up to $10 \mathrm{~ms}$, as compared to the equivalent DMA time range of 5-530 ms inverse frequency. This means that the NMR master curves are necessarily constructed for a much higher shifttransition temperature at which the spread between the segmental/Rouse part and the sticky part is much smallerfinally explaining the much smaller apparent (!) sticky entanglement times in Table 2. It is noted that the forced power-law fit over a rather narrow time interval in the NMR case provides an intrinsic bias for the transition between the two shifting regimes, rather than having it under external control.

III.X. Direct Comparison of NMR and DMA Results. In Figure $9 \mathrm{c}$, we provide a comparison of $\tau_{\mathrm{e}}^{*}$ NMR and $\tau_{\mathrm{e}}^{* \text { DMA }}$ on equal footing, that is, for different temperatures at which each method provides a direct measure on its absolute time or frequency scale. We give only one data point each, along with the $T$ dependence obtained from the DMA shift factors (fits in panel (b)). To locate $\tau_{\mathrm{e}}^{* \text { DMA }}$, the same fit as to the master curve was applied to a single isothermal frequency sweep showing the $E^{\prime \prime}$ maximum prominently. For NMR, $\tau_{\mathrm{e}}^{* \mathrm{NMR}}$ marks the exact time at which the piece of the OACF determined for one specific temperature crosses the entanglement level in the center of the fitted time interval (see Figure S6a). As discussed above, the two quantities should coincide at 
least roughly because of the rather narrow interval for sticky Rouse relaxation owing to the similarity of sticker and entanglement densities.

Reassuringly, across all samples, the DMA and NMR results for $\tau_{\mathrm{e}}^{*}$ are in very good mutual agreement in both absolute time scale and activation energy. This represents a first-time demonstration of the coincidence of sticky chain dynamics assessed on the microscopic scale and by macroscopic rheological properties.

III.XI. Microscopic Model for Sticky Dynamics. To explain all of our observations, we suggest the model depicted in Figure 10, which we expect to hold for the general case of sparse, strongly associating cluster-forming stickers. The main riddle to solve is the question why the $T$ dependence of the BDS-based $\tau_{\mathrm{CR}}$ is nearly the same as the one for the chainbased relaxations (compare the dotted and solid lines in Figure 9c) across the sample series, whereas the absolute time scale of $\tau_{\mathrm{CR}}$ (also plotted in Figure 9c) shows the inverse order as compared to all of the other slower processes (including EP and conductivity). This important finding, which is also corroborated by the NMR results, leads us to conclude that the effective sticker lifetime $\tau_{\mathrm{st}}^{*}$ is not primarily influenced by the absolute time scale of some localized motion of the polar sticky group related to ionic clusters (i.e., $\tau_{\mathrm{CR}}$ ) but more prominently by the factors relevant for such groups actually leaving the cluster and their translational motion through the unpolar matrix. As to a potential alternative, we do not believe that stickers can switch between clusters by the transient close approach and collective rearrangement of whole clusters. Their translational motion should be strongly suppressed by the many (order of 40) attached chains.

We shall first consider a hypothesis formulated in the context of eq $1^{18,29,32}$ that $E_{\mathrm{a}}^{\text {st }}=E_{\mathrm{a}}^{\text {app }}-E_{\mathrm{a}}^{\alpha}$ (see Table 2$)$ is dominated by an enthalpic term $\Delta H_{\mathrm{d} / \mathrm{a}}$ governing the association-dissociation equilibrium constant $K_{\mathrm{d} / \mathrm{a}}$ of a sticker. This would imply that the sample with the highest $E_{\mathrm{a}}^{\text {st }}$ (BIIR $+\mathrm{MI}$ ) should have the lowest concentration of free stickers, leading to a correspondingly longer effective sticker lifetime and chain relaxation. ${ }^{18}$ The fact that its $\tau_{\mathrm{CR}}$ is, however, the shortest leads us to conclude that $\tau_{\mathrm{CR}}$ may not be directly

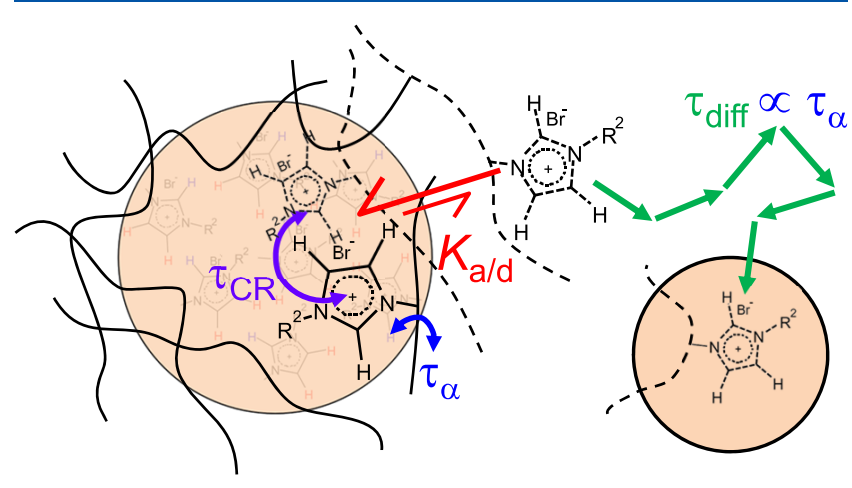

Figure 10. Hierarchy of (thermo)dynamic processes relevant for sticky chain dynamics in cluster-forming systems: segmental process $\left(\tau_{\alpha}\right)$ providing the primary attempt time, cluster relaxation process $\left(\tau_{\mathrm{CR}}\right)$ subject to an internal activation barrier, an associationdissociation equilibrium $\left(K_{\mathrm{a} / \mathrm{d}}\right)$, and finally diffusive motion through the matrix $\left(\tau_{\text {diff }} \propto \tau_{\alpha}\right)$. Note that the underlying $\tau_{\alpha}$ is in our case at least close to the one for the rubber matrix, whereas in ionomers with more polar charged groups at higher concentration, it may be significantly shifted. ${ }^{11,12,14}$ associated with the bare sticker lifetime-it is not even proportional to it. This stands in some contrast to recent results and analyses of a system with hydrogen-bonded pairforming stickers. ${ }^{39}$ We do not have a clear picture of why the $\mathrm{CR}$ in BIIR+MI is the fastest. A dependence on the alkyl tail seems reasonable, with longer and thus more sluggish tails leading to an overall slower cluster relaxation.

It is certainly reasonable to assume that the equilibrium constant $K_{\mathrm{a} / \mathrm{d}}$ for association/dissociation is governed by an enthalpic barrier that follows the same order as $E_{\mathrm{a}}^{\text {st }}$ across the sample series. The dissociated fraction of AI groups, determining the time scale of large-scale relaxation, is certainly larger (corresponding to lower $\Delta H_{\mathrm{d} / \mathrm{a}} \sim E_{\mathrm{a}}^{\mathrm{st}}$ ) for longer alkyl tails due to better compatibility with the matrix. This is also corroborated by previous observations of imidazolium-based ionomers, where longer tails lead to diminished ionic aggregation and larger conductivity. ${ }^{83}$ Note also that $E_{\mathrm{a}}^{\mathrm{app}}$ for $\mathrm{EP}$ and the weak conductivity (see Figure $3 \mathrm{~b}$ ) are about 20-30 $\mathrm{kJ} / \mathrm{mol}$ larger than for the overall chain relaxation, which is in line with the notion that an actual charge carrier (likely an AI group with two $\mathrm{Br}^{-}$counterions) requires a larger dissociation energy due to additional Coulomb interaction.

The ratio $\tau_{\mathrm{e}}^{*} / \tau_{\mathrm{CR}}$ is large and varies between $5 \times 10^{5}$ and 80 for BIIR+MI and $+\mathrm{NI}$, respectively. Associating $\tau_{\mathrm{CR}}$ with $\tau_{\mathrm{st}}^{*}$, this delay was previously explained by (i) the finite return probability of a given dissociated sticker to its original cluster as well as (ii) the long diffusion time $\tau_{\text {diff }}$ to a new cluster (governed by the segmental relaxation of the matrix), both leading to a renormalization of the sticky bond lifetime. ${ }^{18,39}$ Notably, these factors depend only on geometric detailswhich do not vary significantly across the sample series. Thus, the actual sticker lifetime $\tau_{\mathrm{st}}^{*}$ remains elusive. At present, we are not in a position to present a more detailed assessment because existing theoretical approaches either assume densely packed clusters/micelles with negligible return probability ${ }^{29}$ or assume complementary sticker pairs where the sticker dissociation equilibrium also governs the concentration of potential new binding partners. ${ }^{18,39}$ In contrast, sticky clusters can always accept another sticker. A detailed analysis on the basis of a suitably adapted model thus remains to be developed.

III.XII. Effect of Stress on Sticky Chain Relaxation. To investigate the relationship between the sticky chain relaxation and the macroscopic self-recovery behavior, we have performed nonlinear tensile experiments to create a nonequilibrium initial condition, along the lines of similar works performed on cluster-forming telechelic systems ${ }^{28,84}$ or dual networks. ${ }^{9,85}$ In Figure 11, the room-temperature response to the cyclic deformations of the ionic networks is compared with that of the permanent network, using always the same initial strain rate that was lower than or comparable to the rate of chain relaxation between clusters $\left(1 / \tau_{\mathrm{e}}^{*} \mathrm{DMA}\right)$ of all ionic networks. Therefore, strain-induced ionic-cluster rearrangements are expected during the elongation, resulting in much wider hysteresis loops in the ionic samples. For the ionic samples, the maximal stresses reached at around $\epsilon=200 \%$ strain reflect the ionic bond strengths and decrease with the alkyl group length. Thus, at this strain, we indeed expect significant cluster rearrangement. These results appear to be in some disagreement with those of our first publication, ${ }^{3}$ yet in that work an about 10-fold higher deformation rate was used, leading to much less hysteresis in the ionic samples. The observed phenomenon is on the other hand consistent with the strain-rate-dependent hysteresis in hybrid networks, $9,85,86$ 


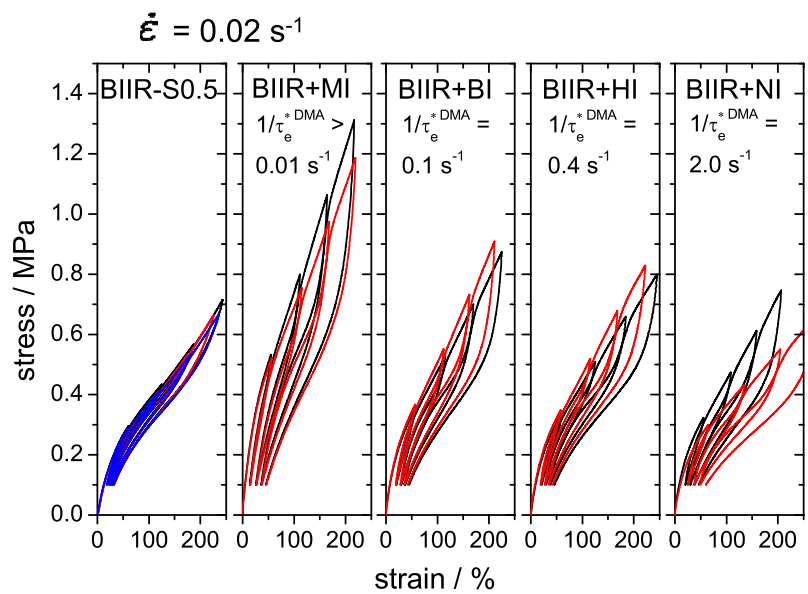

Figure 11. Results of cyclic uniaxial tensile tests at room temperature (different colors denote experiments on different samples illustrating the reproducibility). The sticky chain relaxation rates $1 / \tau_{\mathrm{e}}^{* \text { DMA }}(20$ ${ }^{\circ} \mathrm{C}$ ) are indicated.

where additional permanent cross-links lead to again lower hysteresis at sufficiently slow rates. This emphasizes that future self-healing rubbers based upon the present concept should feature additional permanent cross-links to prevent permanent deformation.

The kinetics of structural reorganizations was further investigated by relaxation experiments; see Figure 12 . The reduced stress $\sigma_{\mathrm{R}}=\sigma /\left(\lambda-\lambda^{-2}\right)(\sigma$ : engineering stress; $\lambda=\epsilon+$ $1)$ is a measure of the normalized stress relative to the prediction of standard rubber elasticity and can be measured

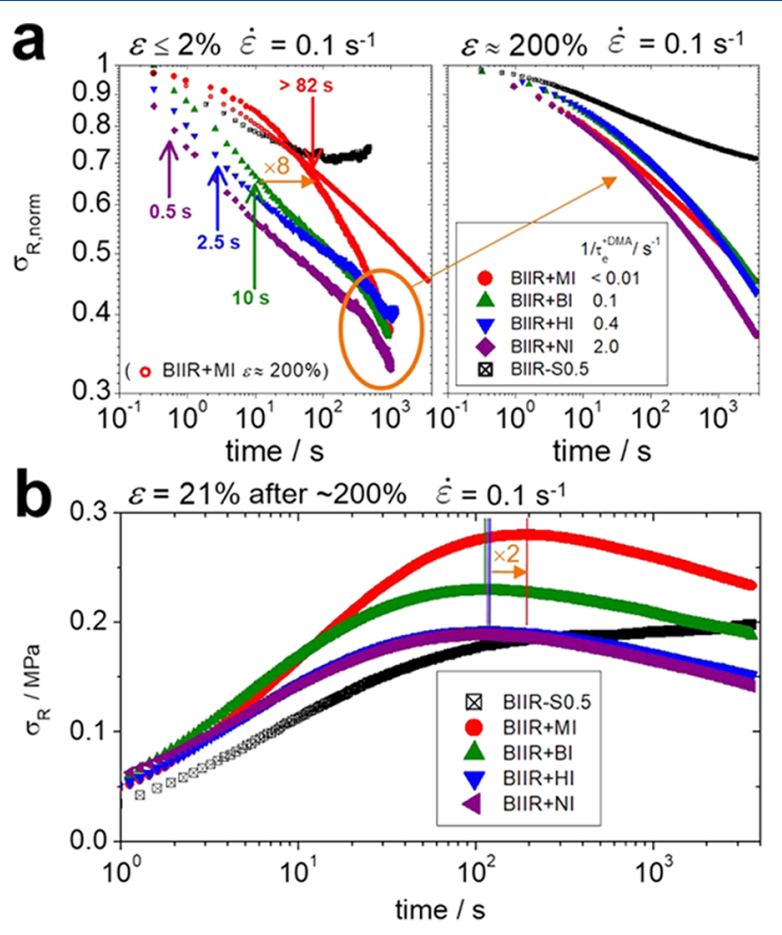

Figure 12. (a) Relaxation of normalized reduced stress at $\leq 2 \%$ (left panel) and $\sim 200 \%$ deformation (right panel) at room temperature. In the left panel, the arrows indicate $\tau_{\mathrm{e}}^{* \text { DMA }}\left(20^{\circ} \mathrm{C}\right)$, and the legend on the right gives their inverse. (b) Recovery and subsequent relaxation of reduced stress after one loading-unloading cycle up to $\epsilon \approx 200 \%$ and back to $21 \%$, with an initial deformation rate $\dot{\epsilon}$ of $\sim 0.1 \mathrm{~s}^{-1}$. Vertical lines mark the transient maxima. for any level of strain. It is a measure of the modulus. In Figure $12 \mathrm{a}$, it was normalized to $\sigma_{\mathrm{R}}(t=0)$ to focus on the relaxation rates. At small strains, BIIR-S0.5 only relaxes at short times (also due to defects), and the long-time plateau is ascribed to the permanent character of the covalent network.

For the ionically cross-linked BIIR, the left panel of Figure 12a demonstrates that the stress relaxation in the linear regime mirrors the DMA results for the sticker lifetime. The right panel provides data for large strain $(\sim 200 \%)$, where for samples BIIR+BI/HI/NI clusters have broken and re-formed due to the low-enough strain rate of $0.1 \mathrm{~s}^{-1}$, which leads to an overemphasis of the terminal relaxation. ${ }^{28}$ In contrast, BIIR + MI did not relax to a comparable degree, which means that it is subject to higher internal stress. Notably, its initial relaxation is now faster than at $2 \%$ elongation; see the open circles in the left panel.

We finally compare the time dependence of the reduced stress recorded after one loading-unloading cycle with the same strain rate separating BIIR+MI from the rest of the samples. All samples were virtually stress-free after unloading to $\epsilon \approx 21 \%$. This enables the detection of a buildup of residual stress, which we compare on the level of absolute reduced stress in Figure $12 \mathrm{~b}$. The initial increase of $\sigma_{\mathrm{R}}$ reflects the recovery of the sample from its forced nonequilibrium state comprising the internal reorganization of sticky clusters and the attached chains and the remaining internal stresses. BIIR $+\mathrm{MI}$ now recovers more quickly and to a higher level due to its comparably larger stored elasticity due to reduced internal reorganization during the initial cycle. The higher transient maximum is even reached only a factor of 2 more slowly instead of the factor of about 8 expected from its linear response. This again shows that, similar to other systems investigated earlier, ${ }^{28,87}$ the effective sticker lifetime decreases upon application of external or internal stress. Thus, the selfhealing capacity of damaged, and thus potentially internally stressed, samples may benefit from this effect.

\section{CONCLUSIONS}

We have presented a comprehensive set of experimental results describing microscopic and macroscopic structural and dynamic properties of lightly charged ionomers based upon butyl rubber with constant density and a systematic variation of the hydrophobicity of the sticky charged alkylimidazolium (AI) sidegroup. SAXS results prove the thermal stability of ionic clusters of about 20 sticky groups in all samples, with a spatial separation governed by their spacing along the chain. Together with further results, we have concluded that the fraction of free stickers is small at all temperatures, that is, a few percent at most.

Our samples do not show any indication of a change in $T_{\mathrm{g}}$ of the matrix or a significant fraction of chains with reduced dynamics, in some contrast to ionomers with higher concentration of charged groups. Proton MAS NMR results focusing on the imidazolium groups indicate a high internal mobility of the ionic clusters, where longer alkyl chains of the AI length lead to a higher amplitude of internal motion. This is corroborated by BDS results, where the relaxation strength of the cluster-related relaxation increases in the same order.

Static proton NMR was used to probe the cross-linked structure and the chain dynamics on the microscopic scale. For the first time, we are able to demonstrate the quantitative agreement of sticky chain relaxation and its macroscopic equivalent probed by DMA. We have found that such a 
comparison is generally challenged when analyzing the related data in terms of apparent master curves. The new concept of a shift-transition temperature was introduced to account for the fact that the breadth of a master curve depends on absolute temperature when the sample dynamics are governed by relaxation processes with different $T$ dependence. The shape of the master curve then depends critically on the actual dynamic window of the technique.

Combining NMR and DMA results with insights from BDS characterizing local and larger-scale dynamics of the polar groups, we have found further evidence for the popular notion that the sticker lifetime depends on both the segmental dynamics (providing the attempt time) and the thermodynamics of the stickers. Thanks to our systematic sample series, we could conclude that the sticky process detected by NMR and DMA is directly related to the BDS-based electrode polarization process and the conductivity. All of these processes thus depend on the time scale of free sticker motion through the unpolar matrix. Contrary to previous assumptions and findings for pair-forming stickers, the BDS-based intracluster relaxation is subject to an activation barrier that appears to be related to the association-dissociation equilibrium of the AI groups (and thus their solubility in the matrix), but its time scale does not appear to correspond to the effective sticker lifetime. A more detailed assessment is currently not possible as a suitable theoretical model is not available.

We hope that our results will stimulate further work aimed at a general understanding of sticky chain dynamics in systems with cluster-forming, associating groups. The studied class of materials has itself proven to be promising for applications as elastomers with self-healing capability.

\section{Corresponding Author}

*E-mail: kay.saalwaechter@physik.uni-halle.de. www.physik. uni-halle.de/nmr.

\section{ORCID}

Frank Böhme: 0000-0001-6128-4658

Ralph H. Colby: 0000-0002-5492-6189

Costantino Creton: 0000-0002-0177-9680

Kay Saalwächter: 0000-0002-6246-4770

\section{ACKNOWLEDGMENTS}

A.M. thanks the members of SIMM/ESPCI in Paris, namely, Juliette Slootman, Ludovic Feige, and Paul Fourton, for their generous support during his research stay. K.S. is indebted to all members of the Colby and Runt research groups during his sabbatical at Penn State University in spring 2018 for their very generous help concerning dielectric, rheological, and SAXS experiments. This includes, but is not limited to, Aijie Han, Liska Utomo, Abigail Fenton, Jiho Seo, Ren Xie, Josh Bostwick, Preeya Kuray, Ciprian Iacob, and Jim Runt. Special thanks are due to Nichole Wonderling for her valuable help in setting up, running, and analyzing the SAXS experiments at the Materials Characterization Lab at PSU. Mario Beiner is thanked for providing additional access to the dielectric spectrometer in Halle. Funding was provided by the DFG through grants SA982/9-1 and BO1121/9-1 in the framework of the Priority Programme SPP 1568 "Design and Generic Principles of Self-Healing Materials.”

\section{REFERENCES}

(1) Brown, H. P.; Gibbs, C. F. Carboxylic Elastomers. Ind. Eng. Chem. 1955, 47, 1006-1012.

(2) Tobolsky, A. V.; Lyons, P. F.; Hata, N. Ionic Clusters in HighStrength Carboxylic Rubbers. Macromolecules 1968, 1, 515-519.

(3) Das, A.; Sallat, A.; Böhme, F.; Suckow, M.; Basu, D.; Wießner, S.; Stöckelhuber, K. W.; Voit, B.; Heinrich, G. Ionic Modification Turns Commercial Rubber into a Self-Healing Material. ACS Appl. Mater. Interfaces 2015, 7, 20623.

(4) Suckow, M.; Mordvinkin, A.; Roy, M.; Singha, N. K.; Heinrich, G.; Voit, B.; Saalwächter, K.; Böhme, F. Tuning the Properties and Self-Healing Behavior of Ionically Modified Poly(isobutylene-coisoprene) Rubber. Macromolecules 2018, 51, 468.

(5) Bagrodia, S.; Wilkes, G. L.; Kennedy, J. P. New PolyisobutyleneBased Model Elastomeric Ionomers: Rheological Behavior. Polym. Eng. Sci. 1986, 26, 662-672.

(6) Poulakis, J. G.; Papaspyrides, C. D. A model process for the recycling of a Surlyn Ionomer. Adv. Polym. Technol. 2000, 19, 203209.

(7) Xavier, T.; Samuel, J.; Kurian, T. Synthesis and Characterization of Novel Melt-Processable Ionomers based on Radiation Induced Styrene Grafted Natural Rubber. Macromol. Mater. Eng. 2001, 286, 507-512.

(8) Zare, P.; Stojanovic, A.; Herbst, F.; Akbarzadeh, J.; Peterlik, H.; Binder, W. H. Hierarchally Nanostructured Polyisobutylene-based Ionic Liquids. Macromolecules 2012, 45, 2074-2084.

(9) Long, R.; Mayumi, K.; Creton, C.; Narita, T.; Hui, C.-U. Time Dependent Behavior of a Dual Cross-Link Self-Healing Gel: Theory and Experiments. Macromolecules 2014, 47, 7243-7250.

(10) Register, R. A.; Prud'homme, R. K. In Ionomers: Synthesis, Structure, Properties and Applications; Tant, M. R., Mauritz, K. A., Wilkes, G. L., Eds.; Springer: Netherlands, 1997; pp 208-256.

(11) Eisenberg, A. Clustering of Ions in Organic Polymers. A Theoretical Approach. Macromolecules 1970, 3, 147-154.

(12) Eisenberg, A.; Hird, B.; Moore, R. B. A New Multiplet-Cluster Model for the Morphology of Random Ionomers. Macromolecules 1990, 23, 4098-4107.

(13) Eisenberg, A.; Kim, J.-S. Introduction to Ionomers; John Wiley \& Sons: New York, 1998.

(14) Malmierca, M. A.; González-Jiménez, A.; Mora-Barrantes, I.; Posadas, P.; Rodríguez, A.; Ibarra, L.; Nogales, A.; Saalwächter, K.; Valentín, J. L. Characterization of Network Structure and Chain Dynamics of Elastomeric Ionomers by Means of ${ }^{1} \mathrm{H}$ Low-Field NMR. Macromolecules 2014, 47, 5655-5667.

(15) Granick, S. Viscoelastic Properties of Sulfonated EthylenePropylene Terpolymer Neutralized with Zinc Cation. J. Appl. Polym. Sci. 1983, 28, 1717-1728. 
(16) Parent, J. S.; Porter, A. M. J.; Kleczek, M. R.; Whitney, R. A. Imidazolium bromide derivatives of poly(isobutylene-co-isoprene): A new class of elastomeric ionomers. Polymer 2011, 52, 5410-5418.

(17) Sallat, A.; Das, A.; Schaber, J.; Scheler, U.; Bhagavatheswaran, E. S.; Stöckelhuber, K. W.; Heinrich, G.; Voit, B.; Böhme, F. Viscoelastic and self-healing behavior of silica filled ionically modified poly(isobutylene-co-isoprene) rubber. $R S C A d v$. 2018, 8, 2679326803.

(18) Stukalin, E. B.; Cai, L.-H.; Kumar, N. A.; Leibler, L.; Rubinstein, M. Self-Healing of Unentangled Polymer Networks with Reversible Bonds. Macromolecules 2013, 46, 7525-7541.

(19) Leibler, L.; Rubinstein, M.; Colby, R. Dynamics of reversible networks. Macromolecules 1991, 24, 4701-4707.

(20) Doi, M.; Edwards, S. F. The Theory of Polymer Dynamics; Oxford University Press: New York, 1986; pp 4701-4707.

(21) Chen, Q.; Zhang, Z.; Colby, R. H. Viscoelasticity of entangled random polystyrene ionomers. J. Rheol. 2016, 60, 1031-1040.

(22) Shivokhin, M. E.; Narita, T.; Talini, L.; Habicht, A.; Seiffert, S.; Indei, T.; Schieber, J. D. Interplay of entanglement and association effects on the dynamics of semidilute solutions of multisticker polymer chains. J. Rheol. 2017, 61, 1231-1241.

(23) Mateyisi, M. J.; Sommer, J.-U.; Müller-Nedebock, K. K.; Heinrich, G. Influence of weak reversible cross-linkers on entangled polymer melt dynamics. J. Chem. Phys. 2018, 148, No. 244901.

(24) Ahmadi, M.; Hawke, L. G. D.; Goldansaz, H.; van Ruymbeke, E. Dynamics of Entangled Linear Supramolecular Chains with Sticky Side Groups: Influence of Hindered Fluctuations. Macromolecules 2015, 48, 7300-7310.

(25) Müller, M.; Dardin, A.; Seidel, U.; Balsamo, V.; Iván, L.; Spiess, H. W.; Stadler, R. Junction Dynamics in Telechelic Hydrogen Bonded Polyisobutylene Networks. Macromolecules 1996, 29, 2577-2583.

(26) Yan, T.; Schröter, K.; Herbst, F.; Binder, W. H.; ThurnAlbrecht, T. Nanostructure and Rheology of Hydrogen-Bonding Telechelic Polymers in the Melt: From Micellar Liquids and Solids to Supramolecular Gels. Macromolecules 2014, 47, 2122-2130.

(27) Goldansaz, H.; Fustin, C.-A.; Wübbenhorst, M.; van Ruymbeke, E. How Supramolecular Assemblies Control Dynamics of Associative Polymers: Toward a General Picture. Macromolecules 2016, 49, 1890-1902.

(28) Yan, T.; Schröter, K.; Herbst, F.; Binder, W. H.; ThurnAlbrecht, T. What Controls the Structure and the Linear and Nonlinear Rheological Properties of Dense, Dynamic Supramolecular Polymer Networks? Macromolecules 2017, 50, 2973-2985.

(29) Semenov, A. N.; Rubinstein, M. Dynamics of Entangled Associating Polymers with Large Aggregates. Macromolecules 2002, 35, 4821-4837.

(30) Amin, D.; Likhtman, A. E.; Wang, Z. Dynamics in Supramolecular Polymer Networks Formed by Associating Telechelic Chains. Macromolecules 2016, 49, 7510-7524.

(31) Colby, R. H.; Zheng, X.; Rafailovich, M. H.; Sokolov, J.; Peiffer, D. G.; Schwarz, S. A.; Strzhemechny, Y.; Nguyen, D. Dynamics of Lightly Sulfonated Polystyrene Ionomers. Phys. Rev. Lett. 1998, 81, 3876-3879.

(32) Rubinstein, M.; Semenov, N. Dynamics of Entangled Solutions of Associating Polymers. Macromolecules 2001, 34, 1058-1068.

(33) Zhang, Z.; Chen, Q.; Colby, R. Dynamics of associative polymers. Soft Matter 2018, 14, 2961-2977.

(34) Ferry, J. D. Viscoelastic Properties of Polymers; John Wiley \& Sons: Toronto, 1980.

(35) Rubinstein, M.; Colby, R. H. Polymer Physics; Oxford University Press: New York, 2003.

(36) Müller, M.; Seidel, U.; Stadler, R. Influence of hydrogen bonding on the viscoelastic properties of thermoreversible networks: analysis of the local complex dynamics. Polymer 1995, 36, 31433150 .

(37) Chen, Q.; Tudryn, G. J.; Colby, R. H. Ionomer dynamics and the sticky Rouse model. J. Rheol. 2013, 57, 1441-1462.
(38) Zhang, Z.; Huang, C.; Weiss, R. A.; Chen, Q. Association energy in strongly associative polymers. J. Rheol. 2017, 61, 11991207.

(39) Gold, B. J.; Hövelmann, C. H.; Lühmann, N.; Székely, N. K.; Pyckhout-Hintzen, W.; Wischnewski, A.; Richter, D. Importance of Compact Random Walks for the Rheology of Transient Networks. ACS Macro Lett. 2017, 6, 73-77.

(40) Gold, B. J.; Hövelmann, C. H.; Lühmann, N.; PyckhoutHintzen, W.; Wischnewski, A.; Richter, D. The microscopic origin of the rheology in supramolecular entangled polymer networks. J. Rheol. 2017, 61, 1211-1226.

(41) Müller, M.; Stadler, R.; Kremer, F.; Williams, G. On the Motional Coupling between Chain and Junction Dynamics in Thermoreversible Networks. Macromolecules 1995, 28, 6942-6949.

(42) Shabbir, A.; Javakhishvili, I.; Cerveny, S.; Hvilsted, S.; Skov, A.; Hassager, O.; Alvarez, N. J. Linear Viscoelsatic and Dielectric Relaxation Response of Unentangled UPy-Based Supramolecular Networks. Macromolecules 2016, 49, 3899-3910.

(43) Trutschel, M.-L.; Mordvinkin, A.; Furtado, F.; Willner, L.; Saalwächter, K. Time-Domain NMR Observation of Entangled Polymer Dynamics: Focus on All Tube-Model Regimes, Chain Center, and Matrix Effects. Macromolecules 2018, 51, 4108-4117.

(44) Brown, S. P.; Schnell, I.; Brand, J. D.; Müllen, K.; Spiess, H. W. The competing effects of $\pi-\pi$ packing and hydrogen bonding in a hexabenzocoronene carboxylic acid derivative: $\mathrm{A}^{1} \mathrm{H}$ solid-state MAS NMR investigation. Phys. Chem. Chem. Phys. 2000, 2, 1735-1745.

(45) de Gennes, P. G. Reptation of a Polymer Chain in the Presence of Fixed Obstacles. J. Chem. Phys. 1971, 55, 572-579.

(46) Ball, R. C.; Callaghan, P. T.; Samulski, E. T. A simplified approach to the interpretation of nuclear spin correlations in entangled polymeric liquids. J. Chem. Phys. 1997, 106, 7352-7361.

(47) Chávez, F. V.; Saalwächter, K. NMR Observation of Entangled Polymer Dynamics: Tube Model Predictions and Constrained Release. Phys. Rev. Lett. 2010, 104, No. 198305.

(48) Vaca Chávez, F.; Saalwächter, K. Time-Domain NMR Observation of Entangled Polymer Dynamics: Universal Behavior of Flexible Homopolymers and Applicability of the Tube Model. Macromolecules 2011, 44, 1549-1559.

(49) Herrmann, A.; Kresse, B.; Gmeiner, J.; Privalov, A. F.; Kruk, D.; Fujara, F.; Rössler, E. A. Protracted Crossover to Reptation Dynamics: A Field Cycling ${ }^{1} \mathrm{H}$ NMR Study Including Extremely Low Frequencies. Macromolecules 2012, 45, 1408-1416.

(50) Brown, S. P.; Spiess, H. W. Advanced Solid-State NMR Methods for the Elucidation of Structure and Dynamics of Molecular, Macromolecular, and Supramolecular Systems. Chem. Rev. 2001, 101, 4125-4156.

(51) Saalwächter, K. Proton multiple-quantum NMR for the study of chain dynamics and structural constraints in polymeric soft materials. Prog. Nucl. Magn. Reson. Spectrosc. 2007, 51, 1-35.

(52) Saalwächter, K. Microstructure and molecular dynamics of elastomers as studied by advanced low-resolution nuclear magnetic resonance methods. Rubber Chem. Technol. 2012, 85, 350-386.

(53) (a) Mordvinkin, A.; Saalwächter, K. Microscopic observation of the segmental orientation autocorrelation function for entangled and constraints polymer chains. J. Chem. Phys. 2017, 146, No. 094902; (b) Mordvinkin, A.; Saalwächter, K. Erratum: "Microscopic observation of the segmental orientation autocorrelation function for entangled and constrained polymer chains" [J. Chem. Phys. 146, 094902 (2017)]. J. Chem. Phys. 2018, 148, No. 089901.

(54) Mark, J. E., Ed. Physical Properties of Polymers Handbook; Springer: New York, 2007.

(55) Maruyama, T.; Nakajima, M.; Ichikawa, S.; Sano, Y.; Nabetani, H.; Furusaki, S.; Seki, M. Small-Angle X-ray Scattering Analysis of Stearic Acid Modified Lipase. Biosci., Biotechnol., Biochem. 2001, 65, 1003-1006.

(56) Kinning, D. J.; Thomas, E. L. Hard-Sphere Interactions between Spherical Domains in Diblock Copolymers. Macromolecules $1984,17,1712-1718$. 

(57) Allen, F. H.; Kennard, O.; Watson, D. G.; Brammer, L.; Orpen,
A. G.; Taylor, R. Tables of Bond Lengths determined by X-Ray and Neutron Diffraction. Part 1. Bond Lengths in Organic Compounds. J. Chem. Soc., Perkin Trans. 2 1987, S1-S19.

(58) Roche, E. J.; Stein, R. S.; Russel, T. P.; MacKnight, W. J. SmallAngle X-Ray Scattering Study of Ionomer Deformation. J. Polym. Sci., Polym. Phys. Ed. 1980, 18, 1497-1512.

(59) MacKnight, W. J.; Earnest, T. R. The Structure and Properties of Ionomers. J. Polym. Sci., Part D: Macromol. Rev. 1981, 16, 41-122.

(60) Handlin, D. L.; MacKnight, W. J.; Thomas, E. L. Critical Evaluation of Electron Microscopy of Ionomers. Macromolecules 1981, 14, 795-801.

(61) Kremer, F., Schönhals, A., Eds. Broadband Dielectric Spectroscopy; Springer: Berlin, 2003.

(62) Richter, D.; Arbe, A.; Colmenero, J.; Monkenbusch, M.; Farago, B.; Faust, R. Molecular Motions in Polyisobutylene: A Neutron Spin Echo and Dielectric Investigation. Macromolecules 1998, 31, 1133-1143.

(63) Kunal, K.; Paluch, M.; Roland, C. M.; Puskas, J. E.; Chen, Y.; Sokolov, A. P. Polyisobutylene: A Most Unusual Polymer. J. Polym. Sci., Part B: Polym. Phys. 2008, 46, 1390-1399.

(64) Wu, J.; Huang, G.; Pan, Q.; Zheng, J.; Zhu, Y.; Wang, B. An investigation on the molecular mobility through the glass transition of chlorinated butyl rubber. Polymer 2007, 48, 7653-7659.

(65) Wu, J.; Huang, G.; Wang, X.; He, X.; Lei, J. Confinement Effect of Polystyrene on the Relaxation Behavior of Polyisobutylene. J. Polym. Sci., Part B: Polym. Phys. 2010, 48, 2165-2172.

(66) Ruan, M.; Yang, D.; Guo, W.; Huang, S.; Wu, Y.; Wang, H.; Wang, H.; Zhang, L. Improved electromechanical properties of brominated butyl rubber filled with modified barium titanate. RSC Adv. 2017, 7, 37148-37157.

(67) Pabst, F.; Gabriel, J.; Weigl, P.; Blochowicz, T. Molecular dynamics of supercooled ionic liquids studied by light scattering and dielectric spectroscopy. Chem. Phys. 2017, 494, 103-110.

(68) Namikawa, H. Characterization of the diffusion process in oxide glasses based on the correlation between electric conduction and dielectric relaxation. J. Non-Cryst. Solids 1975, 18, 173-195.

(69) Sangoro, J. R.; Iacob, C.; Serghei, A.; Friedrich, C.; Kremer, F. Universal scaling of charge transport in glass-forming ionic liquids. Phys. Chem. Chem. Phys. 2009, 11, 913-916.

(70) Mogurampelly, S.; Keith, J. R.; Ganesan, V. Mechanisms Underlying Ion Transport in Polymerized Ionic Liquids. J. Am. Chem. Soc. 2017, 139, 9511-9514.

(71) Saalwächter, K. Robust NMR Approaches for the Determination of Homonuclear Dipole-Dipole Coupling Constants in Studies of Solid Materials and Biomolecules. ChemPhysChem 2013, 14, 3000-3014.

(72) Schäler, K.; Roos, M.; Micke, P.; Golitsyn, Y.; Seidlitz, A.; Thurn-Albrecht, T.; Schneider, H.; Hempel, G.; Saalwächter, K. Basic principles of static proton low-resolution spin diffusion NMR in nanophase-separated materials with mobility contrast. Solid State Nucl. Magn. Reson. 2015, 72, 50-63.

(73) Kim, J.-S.; Eisenberg, A. In Ionomers: Characterization, Theory, and Applications; Schlick, S., Ed.; CRC Press: Boca Raton, 1996; pp $7-31$.

(74) Baum, J.; Pines, A. NMR Studies of Clustering in Solids. J. Am. Chem. Soc. 1986, 108, 7447-7454.

(75) Saalwächter, K.; Lange, F.; Matyjaszewki, K.; Huang, C.-F.; Graf, R. BaBa-xy16: Robust and broadband homonuclear DQ recoupling for applications in rigid and soft solids up to the highest MAS frequencies. J. Magn. Reson. 2011, 212, 204-215.

(76) Chassé, W.; Valentín, J. L.; Genesky, G. D.; Cohen, C.; Saalwächter, K. Precise dipolar coupling constant distribution analysis in proton multiple-quantum NMR of elastomers. J. Chem. Phys. 2011, 134, No. 044907.

(77) Jakisch, L.; Garaleh, M.; Schäfer, M.; Mordvinkin, A.; Saalwächter, K.; Böhme, F. Synthesis and Structural NMR Characterization of Novel PPG/PCL Conetworks Based upon Heterocomple- mentary Coupling Reactions. Macromol. Chem. Phys. 2017, 219, No. 1700327.

(78) Arduengo, A. J., III; Harlow, R. L.; Kline, M. A Stable Crystalline Carbene. J. Am. Chem. Soc. 1991, 113, 361-363.

(79) Chiou, J. Y. Z.; Chen, J. N.; Lei, J. S.; Lin, I. J. B. Ionic liquid crystals of imidazolium salts with a pendant hydroxyl group. J. Mater. Chem. 2006, 16, 2972-2977.

(80) Frenzel, F.; Folikumah, M. Y.; Schulz, M.; Anton, A. M.; Binder, W. H.; Kremer, F. Molecular Dynamics and Charge Transport in Polymeric Polyisobutylene-Based Ionic Liquids. Macromolecules 2016, 49, 2868-2875.

(81) Lang, M.; Sommer, J.-U. Analysis of Entangled Length and Segmental Order Parameter in Polymer Networks. Phys. Rev. Lett. 2010, 104, No. 177801.

(82) Chen, Q.; Huang, C.; Weiss, R. A.; Colby, R. H. Viscoelasticity of Reversible Gelation for Ionomers. Macromolecules 2015, 48, 12211230.

(83) Choi, U. H.; Ye, Y.; Salas de la Cruz, D.; Liu, W.; Winey, K. I.; Elabd, Y. A.; Runt, J.; Colby, R. H. Dielectric and Viscoelastic Responses of Imidazolium-Based Ionomers with Different Counterions and Side Chain Lengths. Macromolecules 2014, 47, 777-790.

(84) Yan, T.; Schröter, K.; Herbst, F.; Binder, W. H.; ThurnAlbrecht, T. Unveiling the molecular mechanism of self-healing in a telechelic, supramolecular polymer network. Sci. Rep. 2016, 6, No. 32356.

(85) Guo, J.; Long, R.; Mayumi, K.; Hui, C.-Y. Mechanics of a Dual Cross-Link Gel with Dynamic Bonds: Steady State Kinetics and Large Deformation Effects. Macromolecules 2016, 49, 3497-3507.

(86) Bai, R.; Yang, J.; Morelle, X. P.; Yang, C.; Suo, Z. Fatigue Fracture of Self-Recovery Hydrogels. ACS Macro Lett. 2018, 7, 312317.

(87) Skrzeszewska, P. J.; Sprakel, J.; de Wolf, F. A.; Fokkink, R.; Cohen Stuart, M. A.; van derGucht, J. Fracture and Self-Healing in a Well-Defined Self-Assembled Polymer Network. Macromolecules 2010, 43, 3542-3548.

(88) It is noted that Figure 1 is somewhat imprecise for the case $N_{\text {st }}$ $\approx N_{\mathrm{e}}$, because the short sticky-Rouse regime is then rather an exponential relaxation. 